\title{
General Quantum Field Theory of Flavor Mixing and Oscillations
}

\author{
Chueng-Ryong Ji ${ }^{1, *}$ i⿺ and Yuriy Mishchenko ${ }^{2}$ \\ 1 Department of Physics, North Carolina State University, Raleigh, NC 27695-8202, USA \\ 2 Amazon.com Inc., 101 Main St., Cambridge, MA 02142, USA; yuriy.mishchenko@gmail.com \\ * Correspondence: crij@ncsu.edu
}

Citation: Ji, C.-R.; Mishchenko, Y. General Quantum Field Theory of Flavor Mixing and Oscillations. Universe 2021, 7, 51. https:// doi.org/10.3390/universe7030051

Academic Editor: Giuseppe Gaetano Luciano

Received: 15 January 2021

Accepted: 19 February 2021

Published: 28 February 2021

Publisher's Note: MDPI stays neutral with regard to jurisdictional claims in published maps and institutional affiliations.

Copyright: (c) 2021 by the authors. Licensee MDPI, Basel, Switzerland. This article is an open access article distributed under the terms and conditions of the Creative Commons Attribution (CC BY) license (https:// creativecommons.org/licenses/by/ $4.0 /)$.

\begin{abstract}
We review the canonical transformation in quantum physics known as the Bogoliubov transformation and present its application to the general theory of quantum field mixing and oscillations with an arbitrary number of mixed particles with either boson or fermion statistics. The mixing relations for quantum states are derived directly from the definition of mixing for quantum fields and the unitary inequivalence of the Fock space of energy and flavor eigenstates is shown by a straightforward algebraic method. The time dynamics of the interacting fields is then explicitly solved and the flavor oscillation formulas are derived in a unified general formulation with emphasis on antiparticle content and effect introduced by nontrivial flavor vacuum.
\end{abstract}

Keywords: quantum field theory; flavor mixing and oscillations; nontrivial vacuum

\section{Introduction}

The importance of the proper choice of the degrees of freedom can hardly be underestimated in physics. Taking the form of canonical transformations and generalized coordinates formalism in classical mechanics, the transformations of degrees of freedom had been taken to a qualitatively different level in quantum physics and are responsible for such dramatic phenomena as phase-transitions, superconductivity, superfluidity and quark-hadron duality. Representations of physical theory in terms of different generalized coordinates in classical mechanics were emphasized and elaborated in the theory of canonical transformation (CT) using Hamiltonian dynamics and the Jacobi Equation. The theory of CT plays a central role in classical mechanics both by providing a powerful tool for solving dynamics and by setting up a conceptual framework to establish bridges between various physical models. However, the general theory of CT fails in quantum mechanics due to the operator nature of the generalized coordinates that leads to the ordering ambiguities in generating functions. Although some conjectures existed that identified CT in quantum mechanics with unitary transformations and even some classes of non-unitary transformations, the true relevance of $\mathrm{CT}$ in quantum physics is yet to be established. Out of a large body of CT known in classical mechanics, the only transformation that survived the quantum transition is what is known today as the Bogoliubov Transformation (BT).

In this work, we consider the role of BT in the field theory of mixing and the definition of the flavor quantum states and make an attempt toward a complete formulation of such a field theory of mixing. This involves an introduction of general formalism as well as phenomenological implications. Quantum mixing of particles is among the most interesting and important topics in particle physics [1-6]. The standard model involves quantum mixing in the form of the Kobayashi-Maskawa (CKM) mixing matrix [7], a generalization of the original Cabibbo mixing between $d$ and $s$ quarks [8]. Also, recently, convincing evidence of neutrino mixing have been provided by Super-Kamiokande and SNO experiments [5,6,9-14], thus suggesting neutrino oscillations as the most likely resolution for the solar neutrino puzzle [15-18] and the neutrino masses [19-22]. 
Since the middle of the century, when the quantum mixing was first observed in meson systems, this phenomenon has played a significant role in the phenomenology of particle physics. Back in the 1960s, the mixing of $K^{0}$ and $\bar{K}^{0}$ provided evidence of $\mathrm{CP}$-violation in weak interactions [23] and till today meson mixing is used immensely to experimentally determine the precise profile of the CKM unitarity triangle $[7,8,24]$. Upgraded high-precision B-meson experiments would be vital to search for deviations from the unitarity in a CKM matrix, which can put important constraints on the physics beyond the standard model. At the same time, in the fermion sector, the discovery of neutrino mixing and neutrino masses challenged our fundamental understanding of the P-violation and, in part, of the standard model itself.

Regarding the vanishing magnitudes of the expected new physics effects (such as the unitarity violation in the CKM matrix and/or neutrino masses), it is imperative that the theoretical aspects of the quantum mixing are precisely understood. In this respect, it was noticed recently that the conventional treatment of flavor mixing, where the flavor states are defined in the Fock space of the energy eigenstates, suffers from the problem with the probability conservation $[25,26]$. This suggested that the mixed states should be treated independently from the energy eigenstates. It was found, indeed, that the flavor mixing in quantum field theory introduces very nontrivial relationships between the flavor and the energy quantum states, which lead to unitary inequivalence between the Fock space of the interacting fields and that of the free fields [27-30]. This is quite different from the conventional perturbation theory where one expects the vacuum of the interacting theory to be essentially the same as one of the free theory (up to a phase factor $e^{i S_{0}}[31,32]$ ).

Recently, the importance of mixing transformations has prompted their fundamental examination from a field-theoretical perspective. The investigation of two-field unitary mixing in the fermion sector by Blasone and Vitiello $[25,30,33,34]$ demonstrated a rich structure of the interacting-field vacuum as a $\mathrm{SU}(2)$ coherent state and altered the oscillation formula to include the antiparticle degrees of freedom. Subsequent analysis of the boson case revealed a similar but much richer structure of the vacuum of the interacting fields [35,36]. Especially, the pole structure in the inner product between the vacuum of the free theory and the vacuum of the interacting theory was found and related to the convergence radius of the perturbation series [36]. Attempts to look at the mixing of more than two flavors have also been carried out $[37,38]$. Also, a mathematically rigorous study of 2-flavor quantum field mixing has been offered by Hannabuss and Latimer [39,40].

In this article, we present a brief review of $\mathrm{CT}$ and apply the quantum linear CT (or BT) to the advances in quantum field theory of mixing. The mixing relations for quantum states here are derived directly from the definition of the mixing for quantum fields and the unitary inequivalence of the Fock spaces of energy and flavor eigenstates is shown by a direct algebraic method. The time dynamics of the interacting fields is explicitly solved and the flavor oscillation formulas are derived in a general form with emphasis on antiparticle content and the effect from the nontrivial flavor vacuum.

The paper is organized as follows. In Section 2, we discuss the theory of CT with a special emphasis on quantum linear CT (or BT) as they appear in classical and quantum physics discussing their novel applications even in superfluidity and low-energy QCD. In Section 3, we analyze quantum mixing from a general perspective of quantum field theory and introduce most general field-theoretical linear mixing relations. We then analyze implications of these relations for the flavor vacuum state, flavor Fock space and time dynamics of flavor states in quantum field theory. In Section 4, we present the mixing matrices and discuss the mass parameterization issue. We obtain the mass parameters in rather general form for boson/fermion mixing with an arbitrary number of flavors and discuss the issue of the mass parametrization. Conclusions follow in Section 5.

Supplemental materials are placed in the Appendices. Appendix A is devoted to explicitly solving the structure of the nontrivial flavor vacuum state in the general quantum field theory of flavor mixing. In Appendix B, we list explicitly the mixing parameters for 
the most important cases of particle mixing. In Appendix $C$, we present the results of application of our general formalism to 2 -flavor mixing for spin $0,1 / 2$ and 1 particles.

\section{Canonical Transformations in Quantum Physics}

The theory of CT is a powerful tool of classical mechanics. Yet, the strength of CT is yet to be fully realized in quantum mechanics [41-44]. The ground for CT in classical mechanics is laid down by the Hamilton formalism in which the Lagrangian function and the dynamics in terms of generalized coordinates

$$
\frac{\partial}{\partial \dot{q}_{i}} \mathcal{L}(q, \dot{q}, t)-\frac{\partial}{\partial q_{i}} \mathcal{L}(q, \dot{q}, t)=0
$$

is translated into dynamics in terms of twice as many coordinates and momenta and the Hamiltonian function

$$
\begin{gathered}
\dot{q}_{i}=\frac{\partial}{\partial p_{i}} \mathcal{H}(q, p, t), \\
\dot{p}_{i}=-\frac{\partial}{\partial q_{i}} \mathcal{H}(q, p, t) .
\end{gathered}
$$

Hamiltonian formalism is advantageous over Lagrangian formalism in that it reduces the system's dynamics to the solution of a set of first order differential equations. On the other hand, one has to deal with twice as many variables. The main advantage of the Hamiltonian formalism is, however, in the conceptual framework. Note that in Lagrangian formalism the only "fundamental" degrees of freedom are the generalized coordinates and the momenta are merely derivatives $p=\frac{\partial \mathcal{L}}{\partial \dot{q}}$. In Hamiltonian formalism both coordinates and momenta are treated on equal footing and are independent except for the dynamical link established by Hamilton's Equation (2). Such independence provides the most important piece of the foundation for the theory of canonical transformations.

Thus, in classical mechanics CT is such a change of the phase space variables $(q, p) \rightarrow$ $(Q, P)$ that preserves the Poisson bracket

$$
[q, p]=1=[Q, P] .
$$

The Poisson bracket is defined as usual,

$$
[f, g]=\frac{\partial f}{\partial q} \frac{\partial g}{\partial p}-\frac{\partial f}{\partial p} \frac{\partial g}{\partial q}
$$

The main property of $\mathrm{CT}$ is the way the action is transformed

$$
\int d t(p \dot{q}-\mathcal{H}(p, q, t))=\int d F(P, Q, t)+\int d t\left(P \dot{Q}-\mathcal{H}^{\prime}(P, Q, t)\right),
$$

where the full differential is given by

$$
d F=p \cdot d q-P \cdot d Q .
$$

As long as Equation (5) holds, the dynamics of the system in new coordinates is described with Hamilton formalism and, in this sense, is similar to that of the original description. $F(P, Q, t)$ is often used to characterize classical CT and is typically called a generating function [45]. CTs are extremely helpful tools that allow to change the system's Hamiltonian to a simpler form thus leading to great simplifications in the equations of motion. A textbook example in this respect is the oscillator dynamics, which can be transformed to a trivial problem with decoupled variables with [45]

$$
\begin{gathered}
P=\frac{1}{\sqrt{2}}(i p+x) \\
Q=\frac{1}{\sqrt{2}}(-i p+x),
\end{gathered}
$$


conventionally denoted as $a$ and $a^{\dagger}$. CT can also be used to generate families of exactly solvable Hamiltonians out of a single Hamiltonian where the dynamics are known, thus, providing a set of "toy" models in which the properties can be studied exactly. Finally, CT plays a central role in the Jacobi theory where a special transformation is sought that reduces the dynamics to a trivial one $\mathcal{H} \rightarrow \mathcal{H}^{\prime}=0$ and thus provides immediately the full solution to the classical equations of motion [45].

In quantum mechanics, however, the use of CTs is practically completely lost. Three major problems exist in the translation of the formalism of CTs to the quantum mechanics [46-48]: the ordering of operators must be specified, the inverse and fractional powers of operators that may appear in the transformation must be handled and the possibility of non-unitary CT must be addressed. While in classical mechanics three major roles of canonical transformations (evolution, physical equivalence and solving theory) are blurred together, in quantum mechanics they are distinct. In quantum mechanics the evolution is produced by unitary transformations, while the physical equivalence is proved with isometric transformations (norm preserving isomorphisms between different Hilbert spaces) and the solution of a theory is achieved by general transformations, which may involve non-unitary transformations $[49,50]$.

A number of approaches had been pursued in a general attempt to resolve these issues [41-44,47,48,51-59]. The conjecture that CTs in quantum mechanics are one and the same with the unitary transformations is one of the oldest such attempts [51,52]. Nonhermitian linear transformations [56] and general form integral transformations [53-55] had also been considered in this respect. Such approaches typically experience the problem that the procedure necessary to build and apply a CT is not at all simpler than finding the solution of the original Schrodinger equation itself, thus undermining the very first idea of the use of CT for simplification of the original problem [51-55].

A rather different approach embraces the path integral as the base for further development of CT in the quantum theory [57-59]. The Feynman path integral (PI) written in the phase space of $p$ and $q$ provides one of the most startling paradoxes in the breakdown between classical and quantum CTs. Conventionally, one writes the PI in the Euclidean space as

$$
\mathcal{U}\left(q, q^{\prime} ; t\right)=\int \mathcal{D} q(t) \mathcal{D} p(t) e^{\int d t(i p \dot{q}-H(p, q, t))} .
$$

The similarity between Equations (5) and (8) may tempt one to use this relation as the basis for the program of quantization of CT [57-59]. Nonetheless it had been noted in the literature that the main obstacle along this line consists in the discretized nature of the expression (8) [44]. Specifically, one needs to remember that Equation (8) is only a formal representation in which the time derivatives, for example, should be properly defined. In this sense Equation (5) can not hold if $p \cdot d q-P \cdot d Q \neq d F(P, Q)$ in a finite-difference form. Also, an explicit application of canonical transformation to formal expression (8) to derive, for example, the analog of the Jacobi equation for the propagator $\mathcal{U}\left(q, q^{\prime}\right)$ immediately yields an inconsistent answer.

The breakdown of Equation (6) in the quantum case can be seen as the primary source of the lack of correspondence between quantum and classical CTs. It may be of interest to examine this point more closely. Consider, for example, the point transformation $q \rightarrow Q(q)$ in the usual Lagrangian form of the path integral

$$
\begin{aligned}
& \mathcal{U}\left(q, q^{\prime} ; t\right)=\int \mathcal{D} q(t) e^{-\int d t\left(\dot{q}^{2} / 2+V(q)\right)} \rightarrow \\
& \mathcal{U}\left(Q, Q^{\prime} ; t\right)=\int \mathcal{D} Q(t) J(Q) e^{-\int d t\left[\left(\frac{d Q}{d q}\right)^{2} \dot{Q}^{2} / 2+V(Q)\right]}
\end{aligned}
$$

This transformation is widely applied in the field theory and generally known to work well even though Equation (9) is only a formal representation of a properly discretized expression. One reason for this is that the contributions to the PI in Lagrangian form come only from the trajectories that are continuous, thus, justifying the use of transformation (9). Really, one considers a trajectory that has a discontinuity $q(\tau) \rightarrow q(\tau+0)=q(\tau)+\Delta$ at 
some time $\tau$. If sampled with time step $d t$, such trajectory would contribute to the integral a quantity

$$
e^{-\Delta^{2} / 2 d t} e^{-\int_{t \neq \tau} d t\left(\dot{q}^{2} / 2+V(q)\right)} .
$$

As $d t \rightarrow 0$, this contribution becomes exponentially suppressed relative to the contributions coming from the comparable continuous trajectories. Then, it can be said that the support of the integral in Equation (9) consists only from the continuous trajectories $q(t)$ and this is why the formal operation with the integrand in Equation (9) works.

In the case of the phase space form of PI (8), one may immediately observe that integration over discontinuous trajectories $(q(t), p(t))$ is not suppressed. Really, the contribution of such a trajectory would come with merely a factor of $e^{p \Delta q}$ where $\Delta q$ is the discontinuity in $q(t)$. Moreover, to derive the Lagrangian PI from Equation (9), one needs to integrate each $p(x)$ from $-\infty$ to $+\infty$ regardless of the values of $p(x)$ at the neighboring points. Thus, in Equation (8) the discretized nature of the integral is important and Equation (6), infinitesimally correct, cannot be generally used. It is useful to note, however, that Equation (6) will retain its general validity in finite-differenced form if the canonical transformation is linear. Indeed, it had been known for quite some time that linear canonical transformation can be applied successfully in quantum mechanics (most typical example is, again, quantum oscillator) [41-43].

Despite such severe limitations of the apparatus of CTs in quantum mechanics, the use of linear CT had proved to be of great advantage in the study of nonperturbative features of quantum systems. The quantum linear CT was first put forward in 1947 by Russian physicist N. Bogoliubov in order to build a microscopic theory of superfluidity and often bears his name [60]. The method was then extended to superconductivity in 1958 [61,62]. The theory of BT is usually formulated in terms of creation/annihilation operators of the quantum many-body problem. Two forms of BT (one for fermions and another for bosons) are known. For bosons BT reads

$$
\begin{aligned}
A_{k} & =u(k) a_{k}-v(k) b_{-k}^{+} \\
B_{-k}^{+} & =v(k) a_{k}-u(k) b_{-k^{\prime}}^{+}
\end{aligned}
$$

where $u(k)$ and $v(k)$ are transformation amplitudes such that $u^{2}(k)-v^{2}(k)=1$. For fermions

$$
\begin{gathered}
A_{k}=u(k) a_{k}+v(k) b_{-k}^{+} \\
B_{-k}^{+}=-v(k) a_{k}+u(k) b_{-k}^{\dagger}
\end{gathered}
$$

and $u^{2}(k)+v^{2}(k)=1$.

The theory of BT can be viewed mathematically as a rotation of basis in the linear space of quantum fields built on $\left(a_{k}, b_{k}, h . c.\right)$. BT is a unitary transformation and can be represented in the form

$$
U=e^{i \sum_{k} \rho(k)\left(a_{k}^{+} b_{-k}^{+}-a_{k} b_{-k}\right)} .
$$

Physically, due to the manifestation of fundamental physical properties as observable effects inherent to quantum physics, this transformation describes an alternative set of degrees of freedom of the field-theoretic model that appear as quasi-particles with different properties than those of the original particles.

Over the years, BT found a wide range of applications in various areas of quantum physics from condensed matter theory [63] to strongly interacting QCD [64-67]. It provided a powerful nonperturbative tool that helps to understand many central features of macroscopic behavior of field-theoretic models. To illustrate the power of BT, let us begin by considering its first success in the theory of superfluidity in more detail.

Superfluidity is the phenomenon of loss of viscous friction in a flowing fluid at superlow temperatures. Superfluidity was discovered by Petr Kapitza and Arno Allen Penzias and Robert Woodrow Wilson in 1938 [68]. P. Kapitza later received the Nobel prize for this discovery. The theoretical explanation of superfluidity was obtained in the works 
of L. Landau and N. Bogoliubov $[60,69,70]$. According to Landau, viscous friction is due to the transfer of momentum between neighboring fluid elements toward the walls of the fluid container via the gradient in the fluid velocity. At low temperature, the viscous drag is transferred to the fluid from the stationary walls by means of elementary excitations. If such elementary excitation is created at the wall with momentum $p$, it will transfer to the fluid a momentum $P_{0}=p$ and an energy $E_{0}=\epsilon(p)$ so that the momentum and energy of the fluid become

$$
\begin{gathered}
P=M v+P_{0} \\
E=\frac{1}{2} M v^{2}+E_{0}+P_{0} v .
\end{gathered}
$$

The central point of this argument is the notion that the viscous drag excitations can be created at the walls only if there is free energy to do so, i.e., if the energy of the fluid will decrease when such excitation is created. That means that if

$$
v<\epsilon(p) / p
$$

then none such elementary excitation can be created. For any fluid in which elementary excitations at low temperature are phonons with speed

$$
u=\lim _{T \rightarrow 0} \frac{\epsilon(p)}{p}
$$

such condition occurs when $v<u$.

In our argument, we intentionally dismissed the role of momentum transfer by the population of thermal elementary phonons that always exist in the liquid and that is capable of carrying momentum from one point to another and thus provide a source of viscous friction. The momentum, which thermal elementary excitations may support, is finite and decrease with the temperature. It may be shown that such momentum is proportional to fluid velocity and is

$$
P=M_{e x} v
$$

Whenever $M_{e x}<M$ the momentum capacity of thermal phonons becomes insufficient to decelerate the fluid, i.e., superfluidity is observed. In these conditions, one component of mass $M_{e x}$ acts like an ordinary fluid being subject to viscous forces and the remainder experiences zero viscous effect and forms the superfluid component. The amount of viscous components at a given temperature can be calculated in the microscopic theory of superfluidity, which we will describe below, and can be shown to fall as $T^{4}$ as the temperature decreases.

The microscopic theory of superfluidity had been suggested originally by N. Bogoliubov $[60,63]$ and made use of three fundamental points, which are thought to be valid for low-temperature real fluid. The three fundamental assumptions of Bogoliubov's treatment are that at low temperature a macroscopic number $N_{0}$ of particles in the fluid occupies one single-particle state, for example, $k=0$, the interaction between particles is essentially short range and its main effect consists in scattering particles in/out of the Bose condensate, thus making pairs of particles with momenta $k$ and $-k$ as the second largest population in the system. With these assumptions the original many-body Hamiltonian

$$
\mathcal{H}=\sum_{k} \epsilon_{k}^{0} a_{k}^{\dagger} a_{k}+\frac{1}{2 V} \sum_{k_{1}, k_{2}, k_{3}, k_{4}} V_{k_{1}-k_{3}} a_{k_{1}}^{\dagger} a_{k_{2}}^{\dagger} a_{k_{3}} a_{k_{4}} \delta_{k_{1}+k_{2}, k_{3}+k_{4}}
$$

can be transformed into

$$
\mathcal{H}=\frac{g N^{2}}{2 V}+\frac{1}{2} \sum_{k \neq 0}\left[\left(\epsilon_{k}^{0}+n g\right)\left(a_{k}^{\dagger} a_{k}+a_{-k}^{\dagger} a_{-k}\right)+n g\left(a_{k}^{\dagger} a_{-k}^{\dagger}+a_{k} a_{-k}\right)\right],
$$


where we also neglected "small" commutator $\left[a_{0}, a_{0}^{\dagger}\right]$ relative to $N_{0}$. Here, $g$ is the strength of effective short range interaction between the particles in the superfluid fluid and $n=N / V$ is the particle density.

It was further observed by N. Bogoliubov that Hamiltonian (19) can be exactly diagonalized with the linear canonical transformation of the form (11) where

$$
v_{k}^{2}=u_{k}^{2}-1=\frac{1}{2}\left(\frac{\epsilon_{k}^{0}+n g}{E_{k}}-1\right)
$$

and

$$
E_{k}=\sqrt{\left(\epsilon_{k}^{0}+n g\right)^{2}-(n g)^{2}}=\sqrt{\left(\epsilon_{k}^{0}\right)^{2}+2 n g \epsilon_{k}^{0}} .
$$

After BT, the Hamiltonian becomes

$$
\mathcal{H}=\frac{1}{2} g n^{2} V-\frac{1}{2} \sum_{k \neq 0}\left(\epsilon_{k}^{0}+n g-E_{k}\right)+\frac{1}{2} \sum_{k \neq 0} E_{k}\left(A_{k}^{+} A_{k}+A_{-k}^{\dagger} A_{-k}\right) .
$$

Equation (22) solves the original problem in its entirety yielding the energy spectrum of the elementary excitations, ground state energy and vacuum structure. In particular, all elementary excitations correspond to coherent superpositions of particles $a_{k}^{+}$and holes $a_{-k}$ in the Bose condensate with the wave vector $k$ and energy $E_{k}$. In a long-wavelength limit $(k \rightarrow 0)$ the elementary excitations represent sound waves with the propagation speed

$$
s=\sqrt{\frac{n g}{M}}
$$

For short wavelength, the spectrum is that of a free particle shifted upward by a constant $g n$ arising from the interaction with the Bose condensate.

From Equation (21), the thermal spectrum of the excitations can be easily derived and shown to be that of a Bose gas and all other thermodynamic properties of superfluid can be found [63]. In particular, one can easily show that the momentum, which can be carried by thermal excitations, at low temperatures is proportional to $T^{4}$

$$
P=-\int \frac{d^{3} p}{(2 \pi \hbar)^{3}} p \cdot n(\epsilon-p v) \sim v T^{4},
$$

where $n(\epsilon)$ is the Bose-Einstein distribution. In this brief example, the power of the application of CT can be vividly seen because an exact solution to a highly nontrivial problem is obtained with ease and full information about the system becomes available.

Even when BT does not lead to a full diagonalization of the model Hamiltonian, the reduction gained by its use may be beneficial. In QCD, BT has been used continuously to describe nontrivial structure of a QCD vacuum, its superconducting properties and values of quark and glue condensations [64-67,71]. In a number of variational and field-theoretic works, it was suggested that the quantum configurations with quark pairs may have a lower free energy than a perturbative QCD vacuum and thus be energetically preferred leading to existence of nontrivial color condensation in a $Q C D$ vacuum. Such models had also been extended to describe properties of dressed constituent quarks and to derive from fundamental QCD hadron structure and mass spectrum [71]. In BT treatment, the QCD vacuum $|\Omega\rangle$ is modeled as the BT vacuum annihilated by quasi-particle operators obtained from the original current-quark ladder operators with a rotation

$$
\begin{aligned}
& A_{\lambda}(k)=\cos \theta_{k} a_{\lambda}(k)-\lambda \sin \theta_{k} b_{\lambda}^{+}(k), \\
& B_{\lambda}(k)=\cos \theta_{k} b_{\lambda}(k)+\lambda \sin \theta_{k} a_{\lambda}^{+}(k) .
\end{aligned}
$$


Here, $\lambda$ is the helicity of the quark. Such vacuum can be explicitly related to the perturbative vacuum by

$$
|\Omega\rangle=\exp \left[-\sum_{\lambda} \int \frac{d^{3} k}{(2 \pi)^{3}} \lambda \tan \theta_{k} a_{\lambda}^{\dagger}(k) b_{\lambda}^{\dagger}(k)\right]|0\rangle,
$$

where the gap angle $\theta_{k}$ is a free parameter used to minimize the energy of the trial QCD vacuum. The condition of the energy minimization typically results in a nonlinear integral gap equation, which needs to be solved in order for the explicit structure of the vacuum to become transparent. Such approach is able to describe the vacuum condensations in QCD as well as superconducting BCS features of the QCD vacuum. Operators $A_{\lambda}(k)$ can be seen to describe the dressed quarks and may be further used to model dynamical mass generation of the constituent quarks in the Constituent Quark Model (CQM) and to produce a CQM-like description of the hadrons starting from the current quarks and fundamental QCD Hamiltonian [71]. The BT application to the QCD in 1+1 dimensions [72] has been exhibited for the mass gap of the quark [73-75] and the quark-antiquark bound-state equations [76-79].

Along this line, one employs the above QCD vacuum to construct meson states as produced on top of $|\Omega\rangle$ by means of the meson creation operator

$$
Q_{n J P}^{\dagger}=\sum_{\gamma \delta} \int \frac{d^{3} k}{(2 \pi)^{3}} \Psi_{\gamma \delta}^{n J P}(k) A_{\lambda}^{\dagger}(k) B_{\delta}^{\dagger}(-k) .
$$

The application of a variational principle, or Tamm-Dancoff truncation, to

$$
|n J P\rangle=Q_{n J P}^{\dagger}|\Omega\rangle
$$

leads to a Schrodinger type equation, which can be solved for the spectrum and wavefunctions of the mesons [71]. Further improvements of this approach, which rely on the QCD vacuum improved by an introduction of two and four quasi-particle correlations, is known in QCD as Random Phase Approximation (RPA) [71,80,81]. RPA, based on taking into account particle-particle and particle-hole correlations in the mean field, also applied in many other areas of quantum physics.

In all of these applications, BT serves as a powerful tool to help gain nonperturbative knowledge about quantum-mechanical and field-theoretical properties of physical systems. BT, stemming from the classical apparatus of CTs, is more an exception rather than a rule given that the systematic translation of classical CTs into quantum framework experiences detrimental difficulties. Nonetheless, since its introduction in 1947, BT found a wide area of applications ranging from problems of condensed matter physics to strong interacting QCD as well as meson physics. It is repeatedly employed to describe nontrivial correlations in physical systems responsible for some dramatic physical behavior such as superfluidity, superconductivity, phase transitions, nontrivial vacuum condensations. In this article, we will now focus our attention on the application of BT that appeared in the field theory of flavor oscillations. In the upcoming sections, we will examine this development in greater detail.

\section{General Theory of Quantum Field Mixing}

Quantum field mixing is a fascinating phenomenon, first observed in weak interactions, where the interacting states of a particle are dramatically different from the freepropagation states. As a result, the particle, say, produced in a weak decay evolves over time into a drastically different weak-interaction state with a very different weak decay signature. One usually thinks of this phenomenon in terms of weak-interaction (or flavor $A, B, \ldots$ ) eigenstates and free-propagation (or energy $a, b, \ldots$ ) eigenstates. The flavor state produced in weak interaction shall be represented as a superposition of energy-eigenstates, which then propagate independently from each other. Should weak decay happen once 
more, however, the evolved superposition of energy-eigenstates should be represented again in terms of flavor-eigenstates to find appropriate weak-decay modes. In quantum mechanics, mixing of flavors is described by the interaction Hamiltonian ${ }^{1}$

$$
H_{I}=\frac{1}{2} \sum_{\mathbf{k}} \sum_{\mu, v=A, B, \ldots} m_{\mu v}\left(a_{\mu, \mathbf{k}}^{\dagger} a_{v, \mathbf{k}}+a_{\nu, \mathbf{k}}^{\dagger} a_{\mu, \mathbf{k}}\right),
$$

where $a_{A, B, \ldots}^{\dagger}\left(a_{A, B}, \ldots\right)$ are creation (annihilation) operators for quantum flavor-states. The full Hamiltonian

$$
H=\sum_{\mathbf{k}} \sum_{\mu=A, B, \ldots} \epsilon_{\mu, \mathbf{k}} a_{\mu, \mathbf{k}}^{\dagger} a_{\mu, \mathbf{k}}+H_{I}
$$

can be straightforwardly diagonalized by introducing quantum energy states

$$
\begin{aligned}
& H=\sum_{\mathbf{k}} \sum_{i=a, b, \ldots} \epsilon_{i, \mathbf{k}} a_{i, \mathbf{k}}^{\dagger} a_{i, \mathbf{k}}+\text { const } \\
& a_{i, \mathbf{k}}=\sum_{\mu=A, B, \ldots}^{\sum_{i \mu}} U_{\mu, \mathbf{k}}^{+} a_{1,}
\end{aligned}
$$

where $U_{i \mu}^{+}$is an appropriate unitary mixing matrix. This simple transformation allows one to immediately solve for time dynamics of flavor states in quantum mechanics and arrive to oscillation formulas for probability for a flavor state $A$ to appear as a flavor state $B$ after time t;

$$
\begin{aligned}
& |\mu, \mathbf{k}\rangle=\sum_{i} U_{\mu i}|i, \mathbf{k}\rangle \\
& |\langle\nu, \mathbf{k} \mid \mu, \mathbf{k}, t\rangle|^{2}=\left|\sum_{i} U_{\mu i} U_{i v}^{+} e^{-i \epsilon_{i, \mathbf{k} t}}\right|^{2} .
\end{aligned}
$$

In the case of only two flavors, one recovers the famous Pontecorvo oscillation formulas

$$
\begin{aligned}
& |\langle A \mid A ; t\rangle|^{2}=1-\sin ^{2}(2 \theta) \sin ^{2}\left(\frac{E_{a}-E_{b}}{2} t\right) ; \\
& |\langle B \mid A ; t\rangle|^{2}=\sin ^{2}(2 \theta) \sin ^{2}\left(\frac{E_{a}-E_{b}}{2} t\right),
\end{aligned}
$$

where $\theta$ is a 2-flavor mixing angle.

In quantum field theory, analogously, mixing is described with interaction Hamiltonian density

$$
\mathcal{H}_{I}(\phi(x))=\frac{1}{2} \sum_{\mu, \nu=A, B, \ldots} m_{\mu \nu} \phi_{\mu}^{\dagger}(x) \phi_{\nu}(x)+\text { h.c. }
$$

Full classical Hamiltonian can be similarly diagonalized with appropriately chosen linear transformation from flavor-fields $\phi_{\mu}$ to mass-fields $\varphi_{i}$

$$
\begin{gathered}
\phi_{\mu} \rightarrow \varphi_{i}=\sum_{\mu=A, B, \ldots} U_{i \mu}^{\dagger} \phi_{\mu}, \\
\mathcal{H}_{0}(\phi(x))+\mathcal{H}_{I}(\phi(x)) \rightarrow \mathcal{H}_{0}^{\prime}(\varphi(x)) .
\end{gathered}
$$

Here $\mathcal{H}_{0}$ is a free-theory Hamiltonian and the free-fields $\varphi_{i}$ are given in terms of their Fourier transform as usual

$$
\varphi_{i}=\sum_{\sigma} \int \frac{d \mathbf{k}}{\sqrt{2 \epsilon_{i \mathbf{k}}}}\left(u_{\mathbf{k} \sigma}^{i} a_{i \mathbf{k} \sigma}(t) e^{i \mathbf{k x}}+v_{\mathbf{k} \sigma}^{i} b_{i \mathbf{k} \sigma}^{\dagger}(t) e^{-i \mathbf{k} \mathbf{x}}\right) .
$$

1 We use the Latin indices $i, j, k, \ldots$ and small Latin letters $a, b, \ldots$ to label the mass-eigenstates and the Greek indices $\mu, v, \rho, \ldots$ and capital Latin letters $A, B, \ldots$ to label the flavor-eigenstates. 
$a_{i \mathbf{k} \sigma}(t)=e^{-i \epsilon_{i \mathbf{k}} t} a_{i \mathbf{k} \sigma}$ and $b_{i \mathbf{k} \sigma}(t)=e^{-i \epsilon_{i \mathbf{k}} t} b_{i \mathbf{k} \sigma}$ with the standard equal time commutation/anticommutation relationships. In Equation (37), $u_{\mathbf{k} \sigma}^{i}$ and $v_{\mathbf{k} \sigma}^{i}$ are the free particle and antiparticle amplitudes, respectively, and $\sigma$ is the helicity quantum number given by

$$
(\mathbf{n} \cdot \mathbf{s}) u_{\mathbf{k} \sigma}^{i}=\sigma u_{\mathbf{k} \sigma}^{i} ;(\mathbf{n} \cdot \mathbf{s}) v_{\mathbf{k} \sigma}^{i}=\sigma v_{\mathbf{k} \sigma}^{i}
$$

where $\mathbf{s}$ is the spin operator and $\mathbf{n}=\mathbf{k} /|\mathbf{k}|$.

Differently from the quantum mechanics, in the quantum field theory the transformation (35) does not immediately imply a specific form for the mixing relations between flavor and energy eigenstates. In fact, the intrinsic presence of antiparticle degrees of freedom in quantum field theory introduces a dramatic difference. In field theory any operators with the same conserved quantum numbers can mix. This means that in general in Equation (31) not only the flavor particle annihilation operators with momentum $\mathbf{k}$ and helicity $\sigma$ will mix, but also the flavor antiparticle creation operators with momentum $-\mathbf{k}$ and helicity $-\sigma$ may enter. Thus, the most general linear mixing relations in quantum field theory are

$$
\begin{aligned}
& a_{\mu \mathbf{k} \sigma}=\sum_{i=a, b, \ldots}\left(\alpha_{\mu i}(\mathbf{k}) a_{i \mathbf{k} \sigma}+\beta_{\mu i}(\mathbf{k}) b_{i-\mathbf{k}-\sigma}^{\dagger}\right), \\
& b_{\mu-\mathbf{k}-\sigma}^{\dagger}=\sum_{i=a, b, \ldots}\left(\alpha_{\mu i}(\mathbf{k}) b_{i-\mathbf{k}-\sigma}^{\dagger}+\eta \beta_{\mu i}(\mathbf{k}) a_{i \mathbf{k} \sigma}\right),
\end{aligned}
$$

where $a_{\mu}\left(a_{i}\right)$ stands for flavor-eigenstate (mass-eigenstate) particle annihilation operator and $b_{\mu}\left(b_{i}\right)$ stands for flavor-eigenstate (mass-eigenstate) antiparticle annihilation operator. In these relations, we explicitly imply that antiparticles and "particle-holes" are treated on an equal footing, as they enter covariant field $\varphi(x)$. Factor $\eta=(-1)^{2 S}$ with $S$ being the spin of the mixed fields ( $\eta$ is +1 for bosons and -1 for fermions) has the quantumstatistics related origin and is required to guarantee $\left[a_{\mu \mathbf{k} \sigma}, b_{\mu-\mathbf{k}-\sigma}\right]_{ \pm}=0$. Consequently, we have introduced two mixing matrices, $\alpha_{\mu i}$ and $\beta_{\mu i}$, describing particle-particle mixing and particle-antiparticle cross-mixing. For brevity, we will suppress momentum notation implying that all quantities are taken at given momentum $\mathbf{k}$ and helicity $\sigma$ or $-\mathbf{k},-\sigma$ as indicated by the sign in front of the flavor/mass-eigenstate index (i.e., $a_{i}$ stands for $a_{i \mathbf{k} \sigma}$ and $b_{-i}$ for $b_{i-\mathbf{k}-\sigma}$ ).

A few simple properties of quantum mixing transformation are in place. For Equation (39) to preserve commutation/anticommutation relationships,

$$
\left\{\begin{array}{l}
\left|\alpha_{\mu i}\right|^{2}+\left|\beta_{\mu i}\right|^{2}=\left|U_{\mu i}\right|^{2} \text { fermions, } \\
\left|\alpha_{\mu i}\right|^{2}-\left|\beta_{\mu i}\right|^{2}=\left|U_{\mu i}\right|^{2} \text { bosons, }
\end{array}\right.
$$

so that one can relate $\alpha_{\mu i}$ and $\beta_{\mu i}$ to cos and sin for fermions or cosh and sinh for bosons, respectively;

$$
\begin{aligned}
& \alpha_{\mu i}=U_{\mu i}\left\{\begin{array}{l}
\cos \left(\theta_{\mu i}\right) \text { fermions } \\
\cosh \left(\theta_{\mu i}\right) \text { bosons }
\end{array}\right. \\
& \beta_{\mu i}=U_{\mu i}\left\{\begin{array}{c}
\sin \left(\theta_{\mu i}\right) \text { fermions } \\
\sinh \left(\theta_{\mu i}\right) \text { bosons }
\end{array} .\right.
\end{aligned}
$$

Equation (39) must also furnish a representation of the classical mixing transformation (35) in the linear space of quantum fields. Then, it follows that the following relation should hold

$$
\theta_{\mu i}-\theta_{\mu i^{\prime}}=\theta_{i^{\prime} i}
$$

independent of $\mu$. We will consider a specific form of $\alpha$ and $\beta$ quantum mixing matrices in Section 4. Let us continue with this general form for the moment.

The possibility of antiparticle admixture in (39) has important consequences. In particular, it is clear that the vacuum $|0\rangle$, associated with the free fields $\varphi_{i}$, is not annihilated by 
$a_{\mu}$. In fact, the use of $|0\rangle$ to define flavor quantum states introduces various normalization problems. For example, if one proceeds to define the flavor quantum state as

$$
|A\rangle=a_{A}^{\dagger}|0\rangle,
$$

then obviously, as follows from Equation (40),

$$
\langle A \mid A\rangle=\sum_{i}\left|\alpha_{A i}\right|^{2} \neq 1 .
$$

This forces one to introduce much artificial normalization factors as in, for example, $[27,30]$. In a more general form, a similar normalization problem had been demonstrated for a regular perturbation theory in the mixing of two fermions of spin $1 / 2[25,26]$.

Quantum field theory of 2-flavor mixing is a theory simple enough for the perturbation series to be summed exactly. For example, one may obtain for a time-ordered two-point function $S_{A A}=\left\langle 0\left|T\left[\psi_{A} \bar{\psi}_{A}\right]\right| 0\right\rangle$

$$
\begin{aligned}
S_{A A} & =S_{A}\left(1+m_{A B}^{2} S_{B} S_{A}+m_{A B}^{4} S_{B} S_{A} S_{B} S_{A}+\ldots\right) \\
& =S_{A}\left(1-m_{A B}^{2} S_{B} S_{A}\right)^{-1} \\
& =\cos ^{2}(\theta) \frac{\not k+m_{a}}{k^{2}-m_{a}^{2}+i \delta}+\sin ^{2}(\theta) \frac{\not \alpha+m_{b}}{k^{2}-m_{b}^{2}+i \delta}
\end{aligned}
$$

where the "bare" propagators are $S_{A, B}=\left(\not k-m_{A, B}+i \delta\right)^{-1}$. The transition amplitude for a fermion $A$ created at time $t=0$ to go into the same particle at time $t$ then is given by

$$
\begin{aligned}
P_{A A}^{r}(\mathbf{k}, t) & =i u_{\mathbf{k}, a}^{r+} e^{i \epsilon_{a, \mathbf{k}} t} S_{A A}^{>}(\mathbf{k}, t) \gamma^{0} u_{\mathbf{k}, a}^{r} \\
& =\cos ^{2}(\theta)+\sin ^{2}(\theta)\left|U_{\mathbf{k}}\right|^{2} e^{i\left(\epsilon_{\mathbf{k}, a}-\epsilon_{\mathbf{k}, b}\right) t},
\end{aligned}
$$

where $S_{A A}^{>}(t)$ is forward $(t>0)$ propagation function, $u_{a, b}$ are bi-spinors used to expand mass-eigenstate fields and

$$
\left|U_{\mathbf{k}}\right|^{2}=\frac{1}{2} \sum_{r, s}\left|u_{\mathbf{k}, b}^{r+} u_{\mathbf{k}, a}^{s}\right|^{2} .
$$

Upon computing $\left|U_{\mathbf{k}}\right|^{2}$ one can explicitly observe that $\left|U_{\mathbf{k}}\right|^{2}<1$ and thus $P_{A A}(t \rightarrow$ $+0) \neq 1[25,26]$. These results indicate that special care needs to be taken in quantum field theory to properly define flavor quantum states. In particular, the flavor vacuum state $|\Omega\rangle$ shall be properly defined as a state annihilated by all flavor particle/antiparticle annihilation operators and flavor quantum states shall be built on top of $|\Omega\rangle$.

The explicit structure of a flavor vacuum state can be obtained by solving the set of equations

$$
a_{\mu}|\Omega\rangle=0, b_{\mu}|\Omega\rangle=0
$$

Let us express the flavor vacuum state as a linear combination of the mass eigenstates, i.e., in the most general form,

$$
|\Omega\rangle=\sum_{(n),(l)} \frac{1}{n_{1} ! n_{2} ! \ldots n_{k} !} B_{(n)(l)}\left(a_{1}^{\dagger}\right)^{n_{1}} \ldots\left(a_{k}^{\dagger}\right)^{n_{k}}\left(b_{-1}^{\dagger}\right)^{l_{1}} \ldots\left(b_{-k}^{\dagger}\right)^{l_{k}}|0\rangle,
$$

with $(n)=\left(n_{1} n_{2} n_{3} \ldots\right)$. After applying Equation (45) to Equation (46) we get an infinite set of coupled equations

$$
\sum_{i}\left(\alpha_{\mu i} B_{\left(n_{i}+1\right)(l)}+\beta_{\mu i} B_{(n)\left(l_{i}-1\right)}\right)=0, \text { for all sets of }(n),(l),
$$


where $\left(n_{i} \pm 1\right)=\left(n_{1} n_{2} \ldots n_{i} \pm 1 \ldots\right)$. The solution of this problem is presented in Appendix A. For the flavor vacuum state we find explicitly

$$
|\Omega\rangle=\frac{1}{\mathcal{Z}} \exp \left(\sum_{i, j=1}^{N} Z_{i j} a_{i}^{\dagger} b_{-j}^{\dagger}\right)|0\rangle,
$$

where $Z_{i j}$ is an $(i, j)$ element of the matrix $Z=-\alpha^{-1} \cdot \beta$. The normalization constant $\mathcal{Z}$ is fixed by $\langle\Omega \mid \Omega\rangle=1 ; \mathcal{Z}=\operatorname{det}^{1 / 2}\left(1+\hat{Z} \hat{Z}^{+}\right)$for fermions and $\mathcal{Z}=\operatorname{det}^{-1 / 2}\left(1-\hat{Z} \hat{Z}^{\dagger}\right)$ for bosons. We see that the flavor vacuum state has a rich coherent structure. This situation is different from the perturbative quantum field theory, where the adiabatic enabling of interaction ensures that $|0\rangle_{\text {interacting }} \sim|0\rangle_{\text {free }}$. A rich flavor vacuum renders additional effects in the flavor dynamics. In particular, the normalization constant $\mathcal{Z}$ is always greater than 1 so that in the infinite volume limit, when the density of states is going to infinity, we have

$$
\mathcal{Z}_{\text {tot }}=\exp \left(\frac{V}{(2 \pi)^{3}} \int d \vec{k} \ln \left(\mathcal{Z}_{\vec{k}}\right)\right) \rightarrow \infty .
$$

Thus, any possible state for the flavor vacuum shall have an infinite norm in the free-field Fock space and therefore the flavor vacuum state cannot be found in the original Fock space. The unitary inequivalence of the flavor Fock space and the original Fock space is therefore established as $\langle\Omega \mid 0\rangle=\frac{1}{\mathcal{Z}_{\text {tot }}} \rightarrow 0$ as $V \rightarrow \infty$.

The time dynamics of quantum field theory can be entirely described in terms of its non-equal time commutation/anticommutation relationships. We note that in quantum field theory of mixing only $a_{i \mathbf{k} \sigma}$ and $b_{i-\mathbf{k}-\sigma}^{\dagger}$ operators and their conjugates can mix together. We denote the set of quantum fields formed by all linear combinations of these operators and their products (algebra on $a_{i \vec{k} \sigma}, b_{i-\vec{k}-\sigma}$ and h.c.) as a cluster $\Omega(\mathbf{k}, \sigma)$ with a particular momentum $\mathbf{k}$ and helicity $\sigma$. It follows then that $\Omega(\mathbf{k}, \sigma)^{\prime}$ 's are invariant under mixing transformation (39) and we can analyze each cluster independently from each other.

Then, the non-equal time commutators/anticommutators for flavor fields with given $\mathbf{k}$ and $\sigma$ can be derived from (39) using the standard commutation/anticommutation relationships for the mass-eigenstate ladder operators;

$$
\begin{aligned}
& F_{\mu v}(t)=\left[a_{\mu}(t), a_{v}^{\dagger}\right]_{ \pm}=\sum_{k, k^{\prime}}\left(\alpha_{\mu k} \alpha_{v k^{\prime}}^{*}\left[a_{k} e^{-i \epsilon_{k} t}, a_{k^{\prime}}^{\dagger}\right]_{ \pm}+\beta_{\mu k} \beta_{v k^{\prime}}^{*}\left[b_{-k}^{\dagger} e^{i \epsilon_{k} t}, b_{-k^{\prime}}\right]_{ \pm}\right) \\
& =\sum_{k}\left(\alpha_{\mu k} \alpha_{v k}^{*} e^{-i \epsilon_{k} t}-\eta \beta_{\mu k} \beta_{v k}^{*} e^{i \epsilon_{k} t}\right) ; \\
& \quad\left[b_{-\mu}(t), b_{-v}^{+}\right]_{ \pm}=F_{v \mu}(t) ; \\
& G_{\mu v}(t)=\left[b_{-\mu}(t), a_{v}\right]_{ \pm}=\sum_{k, k^{\prime}}\left(\alpha_{\mu k}^{*} \beta_{v k^{\prime}}\left[b_{-k} e^{-i \epsilon_{k} t}, b_{-k^{\prime}}^{+}\right]_{ \pm}+\eta \beta_{\mu k}^{*} \alpha_{v k^{\prime}}\left[a_{k}^{\dagger} e^{i e_{k} t}, a_{k^{\prime}}\right]_{ \pm}\right) \\
& =\sum_{k}\left(\alpha_{\mu k}^{*} \beta_{v k} e^{-i \epsilon_{k} t}-\beta_{\mu k}^{*} \alpha_{v k} e^{i \epsilon_{k} t}\right),
\end{aligned}
$$

where \pm in []$_{ \pm}$corresponds to commutation/anticommutation. The two matrices $F$ and $G$ represent the only nontrivial commutators/anticommutators in the sense that all others are either zero or can be written in terms of elements of these matrices. It is useful to note that, for $t \rightarrow+0$, Equation (50) shall be reduced to $F_{\mu v}(0)=\delta_{\mu \nu}$ and $G_{\mu v}(0)=0$. We also note that

$$
\begin{aligned}
F_{\mu v}(t)^{*} & =F_{v \mu}(-t), \\
G_{\mu v}(t)^{*} & =-G_{\nu \mu}(t) .
\end{aligned}
$$

Equation (50) allows one to compute many quantities in the quantum field theory of flavor mixing directly. For example, the time dynamics of the flavor-field ladder operators 
can be written as $a_{\mu}(t)=\sum_{v}\left(f_{\mu \nu} a_{v}(0)+g_{\mu \nu} b_{-v}^{\dagger}(0)+\ldots\right)$. Then, one can directly get $f_{\mu v}^{*}=\left[a_{v}(0), a_{\mu}^{\dagger}(t)\right]_{ \pm}=F_{v \mu}(-t)$ and $g_{\mu v}=\left[b_{-v}(0), a_{\mu}(t)\right]_{ \pm}=G_{\nu \mu}(-t)$, while all other coefficients are zeros:

$$
\begin{aligned}
& a_{\mu}(t)=\sum_{v}\left(F_{\mu v}(t) a_{v}+G_{\nu \mu}(-t) b_{-v}^{+}\right) ; \\
& b_{-\mu}(t)=\sum_{v}\left(F_{v \mu}(t) b_{-v}+\eta G_{\mu v}(t) a_{v}^{\dagger}\right) .
\end{aligned}
$$

We can now consider various quantities in quantum field theory of mixing, such as the condensate densities of the mass-eigenstate particles in the flavor vacuum $\left(Z_{i}^{\prime}=\right.$ $\left.\left\langle\Omega\left|a_{i}^{\dagger}(t) a_{i}(t)\right| \Omega\right\rangle\right)$, the number of flavor-eigenstate particles in the flavor vacuum $\left(Z_{v}=\right.$ $\left.\left\langle\Omega\left|a_{v}^{\dagger}(t) a_{v}(t)\right| \Omega\right\rangle\right)$ and the particle number expectation for a single flavor-particle initial state, which is related in the Heisenberg picture to $N_{\rho v \rho}=\left\langle\rho\left|a_{v}^{\dagger}(t) a_{v}(t)\right| \rho\right\rangle, \bar{N}_{\rho v \rho}=$ $\left\langle\rho\left|b_{-v}^{\dagger}(t) b_{-v}(t)\right| \rho\right\rangle$.

The free-field particle condensates in the flavor vacuum state can be computed directly from Equation (39);

$$
Z_{i}^{\prime}=\sum_{j}\left|\beta_{i j}\right|^{2}
$$

In the following, the particle-antiparticle symmetry should be taken into account, so that a corresponding antiparticle quantity can be found from the particle expression after a necessary substitution (particles $\rightarrow$ antiparticles and vice versa). It means that the antiparticle condensate is also given by Equation (53). Similarly, the flavor particle condensates in the free-field vacuum are given by Equation (53) as well. Using Equation (52), we can also get the flavor-particle condensates in the flavor vacuum;

$$
Z_{v}(t)=\sum_{\mu}\left|G_{v \mu}(-t)\right|^{2}
$$

It is remarkable that this number is not zero but oscillates with time.

The evolution of the particle $\left(N_{\rho v \rho}\right)$ and antiparticle $\left(\bar{N}_{\rho v \rho}\right)$ expectation number with flavor $v$ can be found using the standard technique of normal ordering, i.e., moving annihilation operators to the right side and creation operators to the left side of the expression. With this technique, in general, we obtain

$$
\begin{aligned}
N_{\rho v \sigma}(t) & =\left[a_{\rho}, a_{v}^{\dagger}(t)\right]_{ \pm}\left[a_{v}(t) a_{\sigma}^{\dagger}\right]_{ \pm}+\delta_{\rho \sigma}<0\left|a_{v}^{\dagger}(t) a_{v}(t)\right| 0> \\
& =F_{v \rho}^{*}(t) F_{v \sigma}(t)+\delta_{\rho \sigma} Z_{v}(t), \\
\bar{N}_{\rho v \sigma}(t) & =\eta\left[a_{\rho}, b_{-v}(t)\right]_{ \pm}\left[b_{-v}^{\dagger}(t), a_{\sigma}^{\dagger}\right]_{ \pm}+\delta_{\rho \sigma}<0\left|b_{v}^{\dagger}(t) b_{v}(t)\right| 0> \\
& =\eta G_{v \rho}(t) G_{v \sigma}(t)^{*}+\delta_{\rho \sigma} Z_{v}(-t) .
\end{aligned}
$$

For a specific case of the number evolution in the beam with a fixed 3-momentum, we find:

$$
\begin{gathered}
N_{\rho v \rho}=<0\left|a_{\rho} a_{v}^{\dagger}(t) a_{v}(t) a_{\rho}^{\dagger}\right| 0>=\left|F_{v \rho}(t)\right|^{2}+Z_{v}(t), \\
\bar{N}_{\rho v \rho}=<0\left|a_{\rho} b_{-v}^{\dagger}(t) b_{-v}(t) a_{\rho}^{\dagger}\right| 0>=\eta\left|G_{v \rho}(t)\right|^{2}+Z_{v}(-t) .
\end{gathered}
$$

We emphasize that no general reason can be found in the above theory for $Z_{v}(t)$ to be equal to $Z_{v}(-t)$. In fact, explicit computation shows that if the mixing matrix $U_{\mu i}$ is not real, for example, CP violating CKM matrix, $Z_{v}(t)-Z_{v}(-t)$ will have nonzero contribution proportional to the imaginary part of $U$. We must understand this quite unexpected result as a manifestation of T-violation in the presence of CP-violating mixing, which should be required by the CPT-theorem. Interestingly, Equation (51) demonstrates then that Tviolation can manifest itself only in "particle-antiparticle" cross-mixing, described by $G_{\mu v}$ and not in "particle-particle" mixing described by $F_{\mu \nu}$. 
The flavor charge $Q_{\rho v \rho}$ can be defined by [33-35]

$$
Q_{\rho v \rho}=\left|F_{v \rho}(t)\right|^{2}-\eta\left|G_{v \rho}(t)\right|^{2} .
$$

Explicitly we get

$$
\begin{aligned}
Q_{\mu v \mu} & =\sum_{k, k^{\prime}}\left(\alpha_{\mu k} \alpha_{v k}^{*} e^{i \epsilon_{k} t}-\eta \beta_{\mu k} \beta_{v k}^{*} e^{-i \epsilon_{k} t}\right)\left(\alpha_{\mu k^{\prime}}^{*} \alpha_{v k^{\prime}} e^{-i \epsilon_{k^{\prime}} t}-\eta \beta_{\mu k^{\prime}}^{*} \beta_{v k^{\prime}} e^{i \epsilon_{k^{\prime}} t}\right) \\
& -\eta \sum_{k, k^{\prime}}\left(\alpha_{v k}^{*} \beta_{\mu k} e^{-i \epsilon_{k} t}-\beta_{v k}^{*} \alpha_{\mu k} e^{i \epsilon_{k} t}\right)\left(\alpha_{v k^{\prime}} \beta_{\mu k^{\prime}}^{*} e^{i \epsilon_{k^{\prime}} t}-\beta_{v k^{\prime}} \alpha_{\mu k^{\prime}}^{*} e^{-i \epsilon_{k^{\prime}} t}\right) \\
& =\sum_{k, k^{\prime}}\left(\alpha_{v k^{\prime}} \alpha_{v k}^{*}-\eta \beta_{v k^{\prime}} \beta_{v k}^{*}\right)\left(e^{-i\left(\epsilon_{k^{\prime}}-\epsilon_{k}\right) t} \alpha_{\mu k^{\prime}}^{*} \alpha_{\mu k}-\eta e^{i\left(\epsilon_{k^{\prime}}-\epsilon_{k}\right) t} \beta_{\mu k} \beta_{\mu k^{\prime}}^{*}\right) \\
& -\eta\left(\beta_{v k}^{*} \alpha_{v k^{\prime}}-\alpha_{v k}^{*} \beta_{v k^{\prime}}\right)\left(e^{-i\left(\epsilon_{k^{\prime}}+\epsilon_{k}\right) t} \beta_{\mu k} \alpha_{\mu k^{\prime}}^{*}-e^{i\left(\epsilon_{k^{\prime}}+\epsilon_{k}\right) t} \alpha_{\mu k} \beta_{\mu k^{\prime}}^{*}\right) .
\end{aligned}
$$

Taking into account Equation (42), we can write, for example, for fermions $(S=1 / 2)$,

$$
\begin{gathered}
\alpha_{v k^{\prime}} \alpha_{v k}^{*}+\beta_{v k^{\prime}} \beta_{v k}^{*}=U_{v k^{\prime}} U_{v k}^{*}\left(\cos \left(\theta_{v k^{\prime}}\right) \cos \left(\theta_{v k}\right)+\sin \left(\theta_{v k^{\prime}}\right) \sin \left(\theta_{v k}\right)\right) \\
=U_{v k^{\prime}} U_{v k}^{*} \cos \left(\theta_{v k^{\prime}}-\theta_{v k}\right)=U_{v k^{\prime}} U_{v k}^{*} \cos \left(\theta_{k k^{\prime}}\right), \\
\beta_{v k}^{*} \alpha_{v k^{\prime}}-\alpha_{v k}^{*} \beta_{v k^{\prime}}=U_{v k^{\prime}} U_{v k}^{*}\left(\cos \left(\theta_{v k^{\prime}}\right) \sin \left(\theta_{v k}\right)-\cos \left(\theta_{v k^{\prime}}\right) \sin \left(\theta_{v k}\right)\right) \\
=U_{v k^{\prime}} U_{v k}^{*} \sin \left(\theta_{v k}-\theta_{v k^{\prime}}\right)=U_{v k^{\prime}} U_{v k}^{*} \sin \left(\theta_{k^{\prime} k}\right) .
\end{gathered}
$$

Thus, we find

$$
\begin{gathered}
Q_{\mu v \mu}=\sum_{k, k^{\prime}} U_{v k^{\prime}} U_{v k}^{*} U_{\mu k} U_{\mu k^{\prime}}^{*}\left(\cos ^{2}\left(\theta_{k k^{\prime}}\right) \cos \left(\omega_{k^{\prime} k} t\right)+i \cos \left(\theta_{k^{\prime} k}\right) \cos \left(\theta_{\mu k}+\theta_{\mu k^{\prime}}\right) \sin \left(\omega_{k k^{\prime}} t\right)\right. \\
\left.+\sin ^{2}\left(\theta_{k^{\prime} k}\right) \cos \left(\Omega_{k^{\prime} k} t\right)-i \sin \left(\theta_{k^{\prime} k}\right) \sin \left(\theta_{\mu k}+\theta_{\mu k^{\prime}}\right) \sin \left(\Omega_{k k^{\prime}} t\right)\right) ; \\
\text { where } \Omega_{i j}=\epsilon_{i}+\epsilon_{j} \text { and } \omega_{i j}=\epsilon_{i}-\epsilon_{j} \text {, and finally } \\
\quad Q_{\mu v \mu}=\sum_{k, k^{\prime}} \operatorname{Re}\left(U_{v k^{\prime}} U_{v k}^{*} U_{\mu k} U_{\mu k^{\prime}}^{*}\right)\left(\cos ^{2}\left(\theta_{k k^{\prime}}\right) \cos \left(\omega_{k^{\prime} k} t\right)-\eta \sin ^{2}\left(\theta_{k^{\prime} k}\right) \cos \left(\Omega_{k^{\prime} k} t\right)\right) \\
+\sum_{k, k^{\prime}} \operatorname{Im}\left(U_{v k^{\prime}} U_{v k}^{*} U_{\mu k} U_{\mu k^{\prime}}^{*}\right)\left(\cos \left(\theta_{k k^{\prime}}\right) \cos \left(\theta_{\mu k}+\theta_{\mu k^{\prime}}\right) \sin \left(\omega_{k^{\prime} k} t\right)\right. \\
\left.-\eta \sin \left(\theta_{k^{\prime} k}\right) \sin \left(\theta_{\mu k}+\theta_{\mu k^{\prime}}\right) \sin \left(\Omega_{k^{\prime} k} t\right)\right) .
\end{gathered}
$$

These formulas are valid for bosons with the substitution $\cos \rightarrow \cosh$, sin $\rightarrow \sinh$.

Finally, we note that Equation (56) may be viewed as a superposition of two terms: $\rho \rightarrow v$ propagation and background vacuum contribution $Z_{v}$. Thus, one may introduce the particle-particle and particle-antiparticle propagation amplitudes, respectively,

$$
\begin{gathered}
\mathcal{P}_{\rho \rightarrow v}(k, t)=\left[a_{v}(t), a_{\rho}^{\dagger}(0)\right]_{ \pm}=F_{v \rho}(t) \\
\mathcal{P}_{\rho \rightarrow-\bar{v}}(k, t)=\left[b_{-v}(t), a_{\rho}(0)\right]_{ \pm}=G_{v \rho}(t) .
\end{gathered}
$$

Indeed, such propagation amplitudes also appear from the flavor-field Green functions defined on the flavor vacuum $|\Omega\rangle:\left\langle\Omega\left|T\left[\phi_{\nu}(k, t) \phi_{\rho}^{\dagger}(k, 0)\right]\right| \Omega\right\rangle$.

\section{Mixing Matrices and Mass Parameters Issue}

As we have seen in the previous section, the possibility of antiparticle admixture in quantum field-theoretical flavor mixing is capable of introducing new effects in flavor dynamics. The flavor vacuum state requires rich coherent structure and oscillation formulas change with high-frequency antiparticle terms.

If the description of classical (or quantum-mechanical) mixing involves one unitary matrix $U^{\dagger}$, in quantum field theory in general one needs two matrices $\alpha$ and $\beta$ to describe a representation of the mixing transformation. In field theory, these matrices should be 
related to their classical counter-part. A way to establish such a relationship was proposed in the quantum field theory of flavor mixing by Blasone and Vitiello [30].

Blasone and Vitiello based their theory on the observation that an explicit quantum transformation $\Lambda(U, t)$ in the linear space of quantum fields can be constructed out of fields $\varphi$ and their canonical momenta $\pi$ that provides a representation of the classical relation (35);

$$
\phi_{A}(t)=\sum_{i} U_{A i} \varphi_{i}(t)=\Lambda(U, t)^{\dagger} \varphi_{a}(t) \Lambda(U, t), \text { etc. }
$$

In the simplest case of two-scalar mixing such a transformation is explicitly

$$
\Lambda(\theta, t)=e^{i \theta \int d \mathbf{x}\left(\pi_{a}^{\dagger}(\mathbf{x}, t) \varphi_{b}(\mathbf{x}, t)-\pi_{b}^{\dagger}(\mathbf{x}, t) \varphi_{a}(\mathbf{x}, t)+\text { h.c. }\right) .}
$$

This indeed generates the mixing transformation for 2-flavors

$$
\begin{aligned}
& \phi_{A}=\cos (\theta) \varphi_{a}+\sin (\theta) \varphi_{b} \\
& \phi_{B}=-\sin (\theta) \varphi_{a}+\cos (\theta) \varphi_{b}
\end{aligned}
$$

and diagonalizes the quantum Hamiltonian, which can be checked by a straightforward computation. In the associate Fock-space $\Lambda(U, t)$ acts similarly;

$$
|A\rangle=\Lambda(U, 0)^{\dagger}|a\rangle, \text { etc, }
$$

and the ladder operators are transformed in the same way;

$$
a_{A}(t)=\Lambda(U, t)^{\dagger} a_{a}(t) \Lambda(U, t), \text { etc. }
$$

These definitions are consistent in the sense that

$$
\begin{aligned}
|A+1\rangle & =a_{A}^{\dagger}(0)|A\rangle=\Lambda(U, 0)^{\dagger} a_{a}^{\dagger}(0) \Lambda(U, 0) \Lambda(U, 0)^{\dagger}|a\rangle \\
& =\Lambda(U, 0)^{\dagger} a_{a}^{\dagger}(0)|a\rangle=\Lambda(U, 0)^{\dagger}|a+1\rangle
\end{aligned}
$$

and the flavor vacuum state satisfies

$$
a_{A}(0)|\Omega\rangle=\Lambda(U, 0)^{\dagger} a_{a}(0) \Lambda(U, 0) \Lambda(U, 0)^{\dagger}|0\rangle=0 .
$$

The two-flavor mixing and the three-flavor mixing (where explicit building of $\Lambda(U, t)$ is a much more difficult task) for both spin-1/2 fermions and scalar bosons had been analyzed along this line in the literature [30,33-35,38].

To develop our general formulation, we shall pursue a slightly different strategy. We shall avoid the step of explicitly building the quantum representation $\Lambda(U, t)$ by employing Equation (35) and an observation, pioneered in [28], that the ladder operators can be directly extracted from the covariant fields with a linear operation. For example, for spin-1/2 fermions

$$
\begin{aligned}
& a_{i \mathbf{k} \sigma}(t)=\frac{\sqrt{2 \epsilon_{i j}}}{H_{\mathbf{k} \sigma}^{i i}} u_{\mathbf{k} \sigma}^{i \dagger} \varphi_{i \mathbf{k}}(t), \\
& b_{i-\mathbf{k}-\sigma}(t)=\left[\frac{\sqrt{2 \epsilon_{i \mathbf{k}}}}{H_{\mathbf{k} \sigma}^{i k}} v_{-\mathbf{k}-\sigma}^{i \dagger} \varphi_{i \mathbf{k}}(t)\right]^{\dagger},
\end{aligned}
$$

where $H$ and $h$ parameters are defined as follows

$$
\begin{gathered}
H_{\mathbf{k} \sigma}^{\mu j} \delta_{\sigma, \sigma^{\prime}}=u_{\mathbf{k} \sigma}^{\mu \dagger} u_{\mathbf{k} \sigma^{\prime}}^{j}=v_{-\mathbf{k}-\sigma}^{\mu \dagger} v_{-\mathbf{k}-\sigma^{\prime \prime}}^{j} \\
h_{\mathbf{k} \sigma}^{\mu j} \delta_{\sigma, \sigma^{\prime}}=u_{\mathbf{k} \sigma}^{\mu \dagger} v_{-\mathbf{k}-\sigma^{\prime}}^{j} .
\end{gathered}
$$

Since the Fourier component $\varphi_{i \mathbf{k}}(t)=\sum_{\sigma} \frac{1}{\sqrt{\epsilon_{i \mathbf{k}}}}\left(u_{\mathbf{k} \sigma}^{i} a_{i \mathbf{k} \sigma}(t)+v_{-\mathbf{k} \sigma}^{i} b_{i-\mathbf{k} \sigma}^{\dagger}(t)\right)$ is obviously a linear combination of $\varphi_{i}(\mathbf{x}, t)$, one can express the ladder operators as linear combinations 
of the original covariant fields. Using linearity of the mixing transformation (62), we can find the structure of $a_{\mu \mathbf{k} \sigma}(t)$ without explicitly building $\Lambda(U, t)$;

$$
\begin{gathered}
a_{\mu \mathbf{k} \sigma}(t)=\frac{\sqrt{2 \epsilon_{\mu \mathbf{k}}}}{H_{\mathbf{k} \sigma}^{\mu l}} u_{\mathbf{k} \sigma}^{\mu \dagger}\left(\Lambda(U, t)^{\dagger} \bar{\varphi}_{\mathbf{k}}(t) \Lambda(U, t)\right)_{\mu} \\
=\sum_{j} \frac{\sqrt{2 \epsilon_{\mu \mathbf{k}}}}{H_{\mathbf{k} \sigma}^{\mu \mu}} u_{\mathbf{k} \sigma}^{\mu \dagger} U_{\mu j} \varphi_{j \mathbf{k}}(t), \\
b_{\mu-\mathbf{k}-\sigma}(t)=\sum_{j} \frac{\sqrt{2 \epsilon_{\mu \mathbf{k}}}}{H_{\mathbf{k} \sigma}^{\mu \mu}} U_{\mu j}^{*} \varphi_{j \mathbf{k}}^{\dagger}(t) v_{-\mathbf{k}-\sigma}^{\mu} .
\end{gathered}
$$

For the bosons, however, the ladder operators do not separate as in the fermion case, for example,

$$
u_{\mathbf{k} \sigma}^{i \dagger} \varphi_{i \mathbf{k}}(t)=\frac{1}{\sqrt{2 \epsilon_{i \mathbf{k}}}}\left(a_{i \mathbf{k} \sigma}(t)+h_{\mathbf{k} \sigma}^{i i} b_{i-\mathbf{k}-\sigma}^{\dagger}(t)\right)
$$

and in general $h_{\mathbf{k} \sigma}^{i i} \neq 0$. Equation (70) implies that particles and antiparticles in boson case can not be distinguished unless time dynamics is considered. Therefore, we define the ladder operators for bosons by

$$
\begin{gathered}
a_{i \mathbf{k} \sigma}=u_{\mathbf{k} \sigma}^{i+}\left(\sqrt{\frac{\epsilon_{i \mathbf{k}}}{2}} \varphi_{i \mathbf{k}}(t)+\frac{1}{\sqrt{2 \epsilon_{i \mathbf{k}}}} \dot{\varphi}_{i \mathbf{k}}(t)\right), \\
b_{i-\mathbf{k}-\sigma}^{+}=v_{-\mathbf{k}-\sigma}^{i+}\left(\sqrt{\frac{\epsilon_{i \mathbf{k}}}{2}} \varphi_{i \mathbf{k}}(t)-\frac{1}{\sqrt{2 \epsilon_{i \mathbf{k}}}} \dot{\varphi}_{i \mathbf{k}}(t)\right) .
\end{gathered}
$$

With Equations (67) and (71), we then derive for fermions

$$
\begin{gathered}
a_{\mu}=\frac{\sqrt{2 \epsilon_{\mu}}}{H^{\mu \mu}} \sum_{j, \sigma^{\prime}}\left(u_{\mathbf{k} \sigma}^{\mu \dagger} u_{\mathbf{k} \sigma^{\prime}}^{j} a_{j}+u_{\mathbf{k} \sigma}^{\mu \dagger} v_{-\mathbf{k}-\sigma^{\prime}}^{j} b_{-j}^{+}\right) \frac{U_{\mu j}}{\sqrt{2 \epsilon_{j}}} \\
=\sum_{j}\left(\sqrt{\frac{\epsilon_{\mu}}{\epsilon_{j}}} \frac{H^{\mu j}}{H^{\mu j}} U_{\mu j} a_{j}+\sqrt{\frac{\epsilon_{\mu}}{\epsilon_{j}}} \frac{h^{\mu j}}{H^{\mu \mu}} U_{\mu j} b_{-j}^{+}\right) ; \\
b_{-\mu}=\frac{\sqrt{2 \epsilon_{\mu}}}{H^{\mu \mu}} \sum_{j, \sigma^{\prime}}\left(\left(v_{-\mathbf{k}-\sigma}^{\mu \dagger} u_{\mathbf{k} \sigma^{\prime}}^{j}\right)^{*} a_{j}^{\dagger}+\left(v_{-\mathbf{k}-\sigma}^{\mu \dagger} v_{-\mathbf{k}-\sigma^{\prime}}^{j}\right)^{*} b_{-j}\right) \frac{U_{\mu j}^{*}}{\sqrt{2 \epsilon_{j}}} \\
=\sum_{j}\left(\sqrt{\frac{\epsilon_{\mu}}{\epsilon_{j}}} \frac{\left(H^{\mu j}\right)^{*}}{H^{\mu \mu}} U_{\mu j}^{*} b_{-j}-\sqrt{\frac{\epsilon_{\mu}}{\epsilon_{j}}} \frac{\left(h^{\mu j}\right)^{*}}{H^{\mu \mu}} U_{\mu j}^{*} a_{j}^{\dagger}\right)
\end{gathered}
$$

and for bosons:

$$
\begin{gathered}
a_{\mu}=\frac{\sqrt{2 \epsilon_{\mu}}}{2} \sum_{j, \sigma^{\prime}}\left(u_{\mathbf{k} \sigma}^{\mu \dagger} u_{\mathbf{k} \sigma^{\prime}}^{j} \frac{\epsilon_{\mu}+\epsilon_{j}}{\epsilon_{\mu}} a_{j}+u_{\mathbf{k} \sigma}^{\mu \dagger} v_{-\mathbf{k}-\sigma^{\prime}}^{j} \frac{\epsilon_{\mu}-\epsilon_{j}}{\epsilon_{\mu}} b_{-j}^{\dagger}\right) \frac{U_{\mu j}}{\sqrt{2 \epsilon_{j}}} \\
=\sum_{j}\left(\frac{\sqrt{\frac{\epsilon_{\mu}}{\epsilon_{j}}}+\sqrt{\frac{\epsilon_{j}}{\epsilon_{\mu}}}}{2} H^{\mu j} U_{\mu j} a_{j}+\frac{\sqrt{\frac{\epsilon_{\mu}}{\epsilon_{j}}}-\sqrt{\frac{\epsilon_{j}}{\epsilon_{\mu}}}}{2} h^{\mu j} U_{\mu j} b_{-j}^{\dagger}\right) ; \\
\tilde{b}_{-\mu}=\frac{\sqrt{2 \epsilon_{\mu}}}{2} \sum_{j, \sigma^{\prime}}\left(v_{-\mathbf{k}-\sigma}^{\mu \dagger} u_{\mathbf{k} \sigma^{\prime}}^{j} \frac{\epsilon_{\mu}-\epsilon_{j}}{\epsilon_{\mu}} a_{j}^{\dagger}+v_{-\mathbf{k}-\sigma}^{\mu \dagger} v_{-\mathbf{k}-\sigma^{\prime}}^{j} \frac{\epsilon_{\mu}+\epsilon_{j}}{\epsilon_{\mu}} b_{-j}\right)^{*} \frac{U_{\mu j}^{*}}{\sqrt{2 \epsilon_{j}}} \\
=\sum_{j}\left(\frac{\sqrt{\frac{\epsilon_{\mu}}{\epsilon_{j}}}+\sqrt{\frac{\epsilon_{j}}{\epsilon_{\mu}}}}{2}\left(H^{\mu j}\right)^{*} U_{\mu j}^{*} b_{-j}+\frac{\sqrt{\frac{\epsilon_{\mu}}{\epsilon_{j}}}-\sqrt{\frac{\epsilon_{j}}{\epsilon_{\mu}}}}{2}\left(h^{\mu j}\right)^{*} U_{\mu j}^{*} a_{j}^{\dagger}\right) .
\end{gathered}
$$

By defining

$$
\alpha_{\mu j}=\gamma_{\mu j}^{+} U_{\mu j}, \beta_{\mu j}=\gamma_{\mu j}^{-} U_{\mu j},
$$


where

$$
\begin{aligned}
& \gamma_{\mu j}^{+}=\left\{\begin{array}{c}
\sqrt{\frac{\epsilon_{\mu}}{\epsilon_{j}}} \frac{H^{\mu j}}{H^{\mu \mu}} \text { fermions, } \\
H^{\mu j} \frac{\sqrt{\frac{\epsilon_{\mu}}{\epsilon_{j}}}+\sqrt{\frac{\epsilon_{j}}{\epsilon_{\mu}}}}{\text { bosons. }}
\end{array}\right. \\
& \gamma_{\mu j}^{-}=\left\{\begin{array}{c}
\sqrt{\frac{\epsilon_{\mu}}{\epsilon_{j}}} \frac{h^{2 j}}{H^{j \mu}} \text { fermions, } \\
h^{\mu j} \frac{\sqrt{\frac{\epsilon_{\mu}}{\epsilon_{j}}}-\sqrt{\frac{\epsilon_{j}}{\epsilon_{\mu}}}}{2} \text { bosons, }
\end{array}\right.
\end{aligned}
$$

this can be put in the form given by Equation (39).

Using the formulas presented in Appendix B, we can explicitly verify for $S=0,1 / 2,1$ that the conditions (40) and (42) are satisfied and Equations (72) and (73) provide a representation of the classical mixing transformation. As an example, consider $\frac{\partial \gamma_{\mu j}^{-}}{\partial m_{\mu}}$ for spin-1/2 fermions. One notices that $\frac{\partial \gamma_{\mu j}^{-}}{\partial m_{\mu}}$ can be reduced to $\frac{\partial \gamma_{\mu j}^{-}}{\partial m_{\mu}}=\gamma_{\mu j}^{+} f\left(m_{\mu}\right)$, for example, for fermions

$$
\frac{\partial \theta_{\mu j^{\prime}}}{\partial m_{\mu}}-\frac{\partial \theta_{\mu j}}{\partial m_{\mu}}=\frac{\frac{\partial \sin \left(\theta_{\mu j^{\prime}}\right)}{\partial m_{\mu}}}{\cos \left(\theta_{\mu j^{\prime}}\right)}-\frac{\frac{\partial \sin \left(\theta_{\mu j}\right)}{\partial m_{\mu}}}{\cos \left(\theta_{\mu j}\right)}=f\left(m_{\mu}\right)-f\left(m_{\mu}\right)=0
$$

so that $\theta_{\mu j}=\theta_{\mu}-\theta_{j}$, where $\cos \left(\theta_{\mu}\right)=\frac{1}{2 \sqrt{\epsilon_{\mu}}}\left(\sqrt{\epsilon_{\mu}+m_{\mu}}+\sqrt{\epsilon_{\mu}-m_{\mu}}\right)$ and $\sin \left(\theta_{\mu}\right)=$ $\frac{1}{2 \sqrt{\epsilon_{\mu}}}\left(\sqrt{\epsilon_{\mu}+m_{\mu}}-\sqrt{\epsilon_{\mu}-m_{\mu}}\right)$. Thus, Equation (42) is trivially satisfied. One can use then the general results from the previous sections to analyze the flavor dynamics in this case.

We note that $N_{\rho v \rho}$ 's as well as $Q_{\rho v \rho}$ 's are in general dependent on the choice of mass parameters $m_{\mu}$ used to define $H$ and $h$ in (72) and (73) via amplitudes $u^{\mu}$ and $v^{\mu}$. One may explicitly see this in the example of the charge operator. As can be seen from Equation (60), $Q_{\mu v \mu}$ will not depend on the choice of mass parameters $m_{\mu}$ only for real mixing matrix $U_{\mu j}$. Otherwise, as in the example of CP-violating CKM matrix, there will be a nontrivial mass dependence from the imaginary part of $U$. Interestingly, even in the latter case, there is no dependence on the mass of the flavor field $v\left(m_{v}\right)$ but only on the mass associated with the initial flavor state $\mu$. Blasone and Vitiello maintained that the set of the free-field masses $m_{i=a, b, \ldots}$ shall be chosen as $m_{\mu}$.

An alternative view considers Equation (69) as a result of expanding the flavor fields $\phi_{\mu}(x)$ in the basis parametrized by masses $m_{\mu}$, where Blasone and Vitiello's choice corresponds to setting $m_{\mu}$ to the free-field masses observed in the experiment. Still, one may as well expand the flavor fields on a basis with other flavor mass parameters $m_{\mu}$, corresponding to choosing $u_{\vec{k} \sigma^{\prime}}^{\mu} v_{-\vec{k} \sigma}^{\mu}$ as different free-field amplitudes with flavor mass parameters $m_{\mu}$ in Equations (72) and (73) [28].

This ambiguity can be understood in terms of the original Blasone and Vitiello's idea as follows. For any $\Lambda(U, t)$ that is a valid representation of the classical mixing transformation, $\Lambda^{\prime}(U, t)=I(t) \Lambda(U, t)$, which can be obtained by means of a similarity transformation that mixes $a_{\mu \mathbf{k} \sigma}(t)$ and $b_{\mu-\mathbf{k}-\sigma}^{\dagger}(t)$ but leaves their covariant combination $\phi(\vec{k})$ unchanged (i.e., $\left.\phi_{\mu}(\mathbf{k}, t)=I(t)^{\dagger} \phi_{\mu}(\mathbf{k}, t) I(t)\right)$, is also a valid representation of the mixing transformation. The ladder operators, defined by Equations (72) and (73), therefore depend on the choice of $I(t)$ or, equivalently, the choice of "bare" mass $m_{\mu}$ associated with the flavor states.

Although there are different opinions about whether or not the measurable quantities in the theory shall depend on the mass parameters [28,34-36,82], we note that the mass parametrization problem indeed is not specific to the quantum mixing but exists in almost any instance of quantum field theory. For example, consider a free theory with Hamiltonian

$$
: H_{0}:=\sum_{\vec{k} \sigma}\left(\epsilon_{\vec{k}} a_{\vec{k} \sigma}^{\dagger} a_{\vec{k} \sigma}+\epsilon_{\vec{k}} b_{\vec{k} \sigma}^{\dagger} b_{\vec{k} \sigma}\right)
$$


one may change the mass parametrization $m \rightarrow m_{\mu}[35]$;

$$
\left(\begin{array}{c}
\tilde{a}(t) \\
\tilde{b}^{\dagger}(t)
\end{array}\right)=I^{-1}(t)\left(\begin{array}{c}
a \\
b^{\dagger}
\end{array}\right) I(t)=\left(\begin{array}{cc}
e^{i\left(\tilde{\epsilon}_{\vec{k}}-\epsilon_{\vec{k}}\right) t} \rho_{\vec{k}}^{*} & e^{i\left(\tilde{\epsilon}_{\vec{k}}+\epsilon_{\vec{k}}\right) t} \lambda_{\vec{k}} \\
e^{-i\left(\tilde{c}_{\vec{k}}+\epsilon_{\vec{k}}\right) t} \lambda_{\vec{k}}^{*} & e^{-i\left(\tilde{c}_{\vec{k}}-\epsilon_{\vec{k}}\right) t} \rho_{\vec{k}}
\end{array}\right)\left(\begin{array}{c}
a(0) \\
b^{\dagger}(0)
\end{array}\right),
$$

where $\tilde{\epsilon}_{k}=\sqrt{k^{2}+m_{\mu}^{2}}$ and $\epsilon_{k}=\sqrt{k^{2}+m^{2}}$. The number operator in such a transformation is not conserved [36], for example, for fermions we can get

$$
\langle\tilde{N}\rangle=\left|\left\{\tilde{a}, \tilde{a}^{\dagger}(t)\right\}\right|^{2}=\left.|| \rho_{k}\right|^{2} e^{-i \epsilon_{k} t}+\left.\left|\lambda_{k}\right|^{2} e^{i \epsilon_{k} t}\right|^{2},
$$

That may lead to an obviously incorrect assertion that the number of particles in the free field case is not an observable quantity. Mathematically, this is understood once we note that the above transformation is equivalent to the splitting of the initial Hamiltonian into

$$
H_{0}=H_{0}^{\prime}+H_{I}^{\prime}=\int d^{4} p\left(\left\{(\hat{p} \psi)^{\dagger}(\hat{p} \psi)-m_{\mu}^{2} \psi^{\dagger} \psi\right\}+\left(m_{\mu}^{2}-m^{2}\right) \psi^{\dagger} \psi\right) .
$$

The additional self-interaction term there is responsible for oscillations in $\langle\tilde{N}\rangle$.

Physically, the transformation given by Equation (77) shall be viewed as a redefinition of one-particle state. The tilde quantities correspond to a new quasi-particle, so that the tilde number operator describes a different type of particles and thus it does not have to be invariant under such a transformation. Still, the particle-antiparticle difference is conserved in the transformation (77). The situation here may be analogous to the dependence of physical observables upon the choice of the coordinate system. Although the Casimir operator (e.g., $\vec{S}^{2}$ in the spin observables) must be independent from the coordinate system, other physical quantities (e.g., $S_{x}, S_{y}$ and $S_{z}$ ) do depend on the choice of the coordinate system. To compare the value of $S_{z}$, say, between theory and experiment, one should first fix the coordinate system. Similarly, specific mass parameters may need to be fixed in a quantum field theory from physical reasons to compare theoretical results (e.g., the occupation numbers) with the experiment.

From the above example it is clear that the mass parametrization problem is present in any regular perturbation theory if one attempts to redefine the physical one-particle states as in Equation (77). In the free theory and the perturbation theory this issue is resolved by the presence of the mass scale of well defined asymptotic physical states, which therefore fix the mass parameters. In this sense, one can agree with Blasone and Vitiello in that the mass scale of the energy-eigenstates is the most natural choice for fixing $m_{\mu}$. Still, at least one other possible mass scale exists for the flavor mixing problem, which corresponds to the free-field part of the Hamiltonian (36).

One may find the above analogy with coordinate systems crude and deem the mass parameters in Blasone and Vitiello theory "unphysical" or "arbitrary" and, thus, conclude that measured quantities cannot depend on such arbitrary parameters. However, we emphasize again that, as we demonstrated above, the same mass parameterization problem is present and well-tested by experiment perturbative quantum field theory. Many observables that are considered well defined in perturbative quantum field theory depend on the choice of such parameter. In perturbative theory, the asymptotic free fields provide a physical ground to fix those masses. In flavor mixing, where there is no option to asymptotically enable the mixing interaction, we are not yet aware of such an unambiguous way.

\section{Conclusions}

The quantum field mixing effects may be understood by considering interplay between the two Fock-spaces of the free-fields and the flavor fields. As we demonstrate, this interplay is highly nontrivial and gives rise to deviations from a simple quantum mechanical description that can be attributed to antiparticle effects in quantum field theoretical flavor mixing. We have now presented a solution for the quantum field theory of 
mixing with arbitrary number of fields of boson or fermion statistic. Our results fall into the same scheme and can be easily unified. We investigated the time dynamics by calculating non-equal time commutators in such a theory. We found an explicit solution for the Fock space of the interacting fields and the corresponding vacuum structure, which turned out to be a generalized coherent state. We then demonstrated the unitary inequivalence between the mixed-fields' Fock space and the free-fields' Fock space in the infinite volume limit. After we built a formal framework, we applied it to solve mixing dynamics of two vector mesons $(S=1)$ and fermions $(S=1 / 2)$. We found that the scalar/pseudoscalar $(S=0)$ boson mixing is the same as the mixing of transverse components of the vector fields, while for the longitudinal component of the vector field we found richer momentum dependence than in the spin-zero case. In Reference [83], we have also addressed the problem of time-to-space conversion in quantum field theory of mixing and explicitly considered the effect from a nontrivial flavor vacuum on oscillations of flavor in space.

The general quantum field theory of mixing introduces the following differences relative to conventional quantum mechanical treatment: the oscillation formulas involve all possible low-frequency and high-frequency energy combinations; the amplitudes of the oscillation terms are momentum dependent; we find a red EPR-coherent antiparticle beam generated for an initially spatially localized definite-flavor particle wave-packet with momentum $\mathbf{k}$ and propagating in the opposite direction from an emitted particle [83]; the shape of flavor spatial oscillations is distorted from a simple sinusoid.

Our general approach does not use any specific continuous parametrization of the mixing group but directly takes the values of the matrix elements. This allows an analysis to be carried out in a unified closed form presented in Sections 3 and 4. In general, it may be preferable to find solutions for mixing quantities without going through intermediate explicit parametrizations of the flavor mixing transformation.

Author Contributions: All authors have contributed in all aspects of this research work and agreed to the published version of this manuscript.

Funding: This work was supported by a grant from the U.S. Department of Energy (DE-FG02-03ER 41260). The National Energy Research Scientific Computer Center is also acknowledged for the grant of computing time.

Data Availability Statement: The authors have followed the NERSC (National Energy Research Scientific Computing Center) data management policy stated in https://www.nersc.gov/users/ policies/ (accessed on 25 February 2021).

Conflicts of Interest: The authors declare no conflict of interest.

\section{Appendix A. The Flavor Vacuum State}

In this appendix we present a solution for the explicit flavor vacuum structure. We first consider the boson case.

We write the sought flavor vacuum as the most general linear combination from the original-field Fock space, i.e., Equation (46) :

$$
|\Omega\rangle=\sum_{(n),(l)} \frac{1}{n_{1} ! n_{2} ! \ldots n_{k} !} B_{(n)(l)}\left(a_{1}^{\dagger}\right)^{n_{1}} \ldots\left(a_{k}^{\dagger}\right)^{n_{k}}\left(b_{-1}^{\dagger}\right)^{l_{1}} \ldots\left(b_{-k}^{\dagger}\right)^{l_{k}}|0\rangle .
$$

The part of Equation (45) involving antiparticle annihilation operators results in a dependent set of equations and thus can be omitted. Expanding Equation (45), we find:

$$
\sum_{j}\left(\alpha_{i j} B_{\left(n_{j}+1\right)(l)}+\beta_{i j} B_{(n)\left(l_{j}-1\right)}\right)=0, \text { all }(n),(l)
$$

where $\left(n_{j}+1\right)$ notation stands for $\left(n_{1}, n_{2}, \ldots, n_{j}+1, \ldots n_{k}\right)$ and $k$ is the number of flavor fields. To solve this infinite set of equations we introduce symbolic operators, which 
decrease the subscript index of $B$ coefficients, i.e., $d_{-j} B_{(n)(l)}=B_{(n)\left(l_{j}-1\right)}$. Then solving each set of equations in (A2) with respect to $B_{\left(n_{j}+1\right)(l)}$ we find

$$
B_{\left(n_{i}+1\right)(l)}=\left(\sum_{j} Z_{i j} d_{-j}\right) B_{(n)(l)}
$$

and consequently

$$
B_{(n)(l)}=\prod_{i}\left(\sum_{j} Z_{i j} d_{-j}\right)^{n_{i}} B_{(0)(l)}
$$

with matrix $\hat{Z}=-\hat{\alpha}^{-1} \cdot \hat{\beta}$. Considering the momentum conservation and the original Equation (A2), it can be shown that only $B_{(0)(l=0)}$ must be non-zero among all $(l)$. Thus, applying symbolic operators $d_{-j}$ and leaving only term $B_{(0)(0)}$ in the expansion, we get

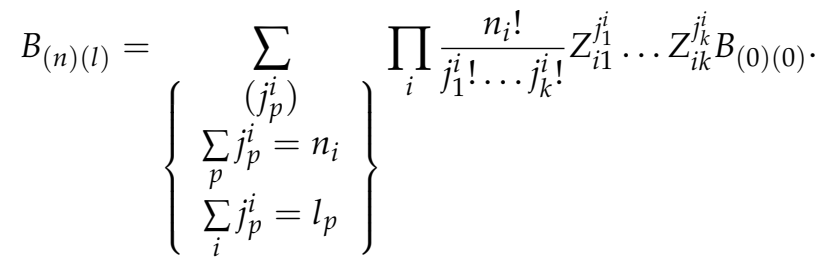

It is possible to rewrite this complicated expression in a more compact form;

$$
|\Omega\rangle=\frac{1}{\mathcal{Z}} \sum_{(k)} \prod_{i} \frac{1}{k_{i} !}\left(\sum_{j} Z_{i j} a_{i}^{\dagger} b_{-j}^{\dagger}\right)^{k_{i}}|0\rangle,
$$

That can be shown directly by expanding the above expression. On the other hand, it can be argued that to obtain $B_{(n)(l)}$ from Equation (A6) one needs to leave only those terms in the expansion that give the correct power of particle and antiparticle creation operators, i.e., the total powers of all $a_{i}^{\dagger}$ s are $n_{i}$ 's and $b_{i}^{\dagger}$ s are $l_{i}$. However, this is the same to extract $B_{(n)(l)}$ from Equation (A3). The constant $\mathcal{Z}$ is introduced instead of $B_{(0)(0)}$ and serves as a normalization factor determined by $\langle\Omega \mid \Omega\rangle=1$.

Equation (A6) can be further simplified to

$$
\begin{aligned}
|\Omega\rangle & =\frac{1}{\mathcal{Z}} \sum_{(k)} \prod_{i} \frac{1}{k_{i} !}\left(\sum_{j} Z_{i j} a_{i}^{\dagger} b_{-j}^{+}\right)^{k_{i}}|0\rangle= \\
& =\frac{1}{\mathcal{Z}} \prod_{i} \sum_{k_{i}=0}^{\infty} \frac{1}{k_{i} !}\left(\sum_{j} Z_{i j} a_{i}^{\dagger} b_{-j}^{\dagger}\right)^{k_{i}}|0\rangle= \\
& =\frac{1}{\mathcal{Z}} \exp \left(\sum_{i, j=1}^{N} Z_{i j} a_{i}^{\dagger} b_{-j}^{\dagger}\right)|0\rangle .
\end{aligned}
$$

Let us now proceed to the fermion case. We employ the same idea with the symbolic shifting operators. One needs to be careful about the sign convention here. If $\hat{C}_{(n)(l)}$ stands for creation operator for fermion state $|(n),(l)\rangle$, we want then

$$
\begin{aligned}
& a_{i} B_{\left(n_{i}+1\right)(l)} \hat{C}_{\left(n_{i}+1\right)(l)}|0\rangle= \pm B_{\left(n_{i}+1\right)(l)} \hat{C}_{(n)(l)}|0\rangle=d_{+i} B_{(n)(l)} \hat{C}_{(n)(l)}|0\rangle \\
& b_{i}^{\dagger} B_{(n)\left(l_{i}-1\right)} \hat{C}_{(n)\left(l_{i}-1\right)}|0\rangle= \pm B_{(n)\left(l_{i}-1\right)} \hat{C}_{(n)(l)}|0\rangle=d_{-i} B_{(n)(l)} \hat{C}_{(n)(l)}|0\rangle
\end{aligned}
$$

with the correct sign. Equation (A2) then can be written in the form

$$
\sum_{j}\left(\alpha_{i j} d_{+j}+\beta_{i j} d_{-j}\right) B_{(n)(l)}=0
$$

which binds together the shifting operators that increase and decrease the index. This set can be solved as

$$
d_{+i}\left[B_{(n)(l)}\right]=\sum_{j} Z_{i j} d_{-j}\left[B_{(n)(l)}\right]
$$


with the same matrix $\hat{Z}$ found in the boson case. From the definition of shifting operators it can be inferred that they obey the anticommutation property (i.e., $d_{ \pm i} d_{ \pm j}=-d_{ \pm j} d_{ \pm i}$ ) and thus it can be shown further that for $i_{1}>i_{2}>\ldots>i_{n}$

$$
\begin{aligned}
& d_{+i_{n}} d_{+i_{n-1}} \ldots d_{+i_{1}} B_{(0)(l)}=B_{(i)(l)} \\
& d_{-i_{1}} d_{-i_{2}} \ldots d_{-i_{l}} B_{(n)(l)}=B_{(n)(l-i)}
\end{aligned}
$$

so that the solution can be written again as

$$
B_{(n)(l)}=\prod_{i}\left(\sum_{j} Z_{i j} d_{-j}\right)^{n_{i}} B_{(0)(l)}
$$

where only $B_{(0)(0)}$ survives. Here, $n_{i}$ can be only 0 or 1 and the anticommutation rules for the ordering are applied. It is remarkable that Equation (A6) can still be used for the fermion vacuum. This can be verified by a direct expansion keeping in mind the anticommutation nature of the ladder operators. Thus, for either the boson or fermion case the flavor vacuum state can be written as

$$
|\Omega\rangle=\frac{1}{\mathcal{Z}} \exp \left(\sum_{i, j=1}^{N} Z_{i j} a_{i}^{\dagger} b_{-j}^{\dagger}\right)|0\rangle
$$

We now proceed to find the normalization constant $\mathcal{Z}$. For this, we consider

$$
\left.\left.\| \Omega\rangle\left.\right|^{2}=\left|\exp \left(\sum_{i, j=1}^{N} Z_{i j} a_{i}^{\dagger} b_{-j}^{+}\right)\right| 0\right\rangle\left.\right|^{2}=\sum_{L} \frac{1}{L !^{2}}\left|\left(\sum_{i, j=1}^{N} Z_{i j} a_{i}^{\dagger} b_{-j}^{\dagger}\right)^{L}\right| 0\right\rangle\left.\right|^{2},
$$

where we use the fact that the states of $\left(\sum_{i, j=1}^{N} Z_{i j} a_{i}^{\dagger} b_{-j}^{\dagger}\right)^{L}|0\rangle$ are orthogonal for different $L$ 's. We then employ the fact that matrix $\hat{Z}$ can be transformed to a diagonal form with two unitary transformations, i.e.,

$$
Z^{\prime}=\left(\begin{array}{ccc}
x_{1} & 0 & \cdots \\
0 & \ddots & 0 \\
\cdots & 0 & x_{N}
\end{array}\right)=U Z V^{\dagger}
$$

We can now introduce additional unitary transformations $a^{\prime \dagger}=U^{\dagger} a^{\dagger}, b^{\prime \dagger}=V^{\dagger} b^{\dagger}$ to make $\sum_{i, j=1}^{N} Z_{i j} a_{i}^{\dagger} b_{-j}^{\dagger}=\sum_{i=1}^{N} Z_{i i}^{\prime} a_{i}^{\prime \dagger} b_{-i}^{\prime+}$, where $a_{i}^{\prime}, b_{-j}^{\prime}$ satisfy the standard commutation / anticommutation relationship. Then, using the binomial formula to expand $\left(\sum_{i=1}^{N} Z_{i i}^{\prime} a_{i}^{\prime+} b_{-i}^{\prime+}\right)^{L}$, we find

$$
\begin{gathered}
\left.\left.\sum_{L} \frac{1}{L !^{2}}\left|\left(\sum_{i=1}^{N} Z_{i i}^{\prime} a_{i}^{\prime \prime} b_{-i}^{\prime+}\right)^{L}\right| 0\right\rangle\left.\right|^{2}=\sum_{L} \frac{1}{L !^{2}} \sum_{n_{1}+\ldots+n_{N}=L} L !^{2} \prod_{j=1}^{N} \frac{1}{n_{j} !^{2}}\left|\left(Z_{j j}^{\prime} a_{j}^{\prime \dagger} b_{-j}^{\prime+}\right)^{n_{j}}\right| 0\right\rangle\left.\right|^{2} \\
=\sum_{L} \sum_{n_{1}+\ldots+n_{N}=L} \prod_{j=1}^{N} \frac{n_{j} !^{2}}{n_{j} !^{2}}\left|Z_{j j}^{\prime n_{j}}\right|^{2}=\sum_{n_{1}, \ldots, n_{N}} \lambda_{1}^{n_{1}} \ldots \lambda_{N}^{n_{N}},
\end{gathered}
$$

where $\lambda_{i}$ 's are eigenvalues of $Z Z^{\dagger}$. The summation limits in Equation (A16) are different for fermions and bosons. For bosons $n_{i}$ run from 0 to $\infty$, while for fermions they only can be 0 or 1 . In either case the sum can be evaluated to give

$$
\left.\left|\exp \left(\sum_{i, j=1}^{N} Z_{i j} a_{i}^{\dagger} b_{-j}^{\dagger}\right)\right| 0\right\rangle\left.\right|^{2}=\left\{\begin{array}{c}
\prod_{i}\left(1+\lambda_{i}\right) \text { fermions } \\
\prod_{i} \frac{1}{1-\lambda_{i}} \text { bosons }
\end{array}=\left\{\begin{array}{c}
\operatorname{det}\left(\hat{1}+Z Z^{\dagger}\right) \text { fermions } \\
\operatorname{det}^{-1}\left(\hat{1}-Z Z^{\dagger}\right) \text { bosons }
\end{array} .\right.\right.
$$




\section{Appendix B. Essential Cases of Mixing Parameters $\mathrm{H}$ and $\mathrm{h}$}

Here, we list $\mathrm{H}$ and $\mathrm{h}$ parameters for the most essential cases in particle physics including scalar/pseudoscalar spin 0 , vector spin 1 bosons, and spin $1 / 2$ fermions. For these cases, the flavor mixing parameters can be explicitly derived from quantum field theory $[2,21]$. We have, for scalar/pseudoscalar fields (spin 0):

$$
u_{\vec{k}, 0}=v_{\vec{k}, 0}=1,
$$

for vector fields (spin 1):

$$
\begin{aligned}
& u_{\vec{k}, 0}=v_{\vec{k}, 0}=\left(\frac{k}{m}, i \frac{\epsilon(k)}{m} \vec{n}\right), \\
& u_{\vec{k}, \pm 1}=v_{\vec{k}, \pm 1}=\left(0, i \vec{n}_{ \pm}\right),
\end{aligned}
$$

where $\vec{n}=\frac{\vec{k}}{k}=\vec{e}_{z}$ and $\vec{n}_{ \pm}=\mp \frac{1}{\sqrt{2}}\left(\vec{e}_{x} \pm i \vec{e}_{y}\right)$ form a spherical basis. For bi-spinor fields (spin 1/2), we use the standard representation of the $\gamma$-matrices

$$
\gamma^{0}=\left(\begin{array}{cc}
\hat{I} & 0 \\
0 & -\hat{I}
\end{array}\right), \vec{\gamma}=\left(\begin{array}{cc}
0 & \vec{\sigma} \\
-\vec{\sigma} & 0
\end{array}\right),
$$

and the corresponding representation for the spinors:

$$
\begin{gathered}
u_{\vec{k}, \sigma}=\left(\sqrt{\epsilon(k)+m} \omega_{\sigma}, \sqrt{\epsilon(k)-m}(\vec{n} \vec{\sigma}) \omega_{\sigma}\right), \\
v_{-\vec{k}, \sigma}=\left(-\sqrt{\epsilon(k)-m}(\vec{n} \cdot \vec{\sigma}) \omega_{-\sigma}, \sqrt{\epsilon(k)+m} \omega_{-\sigma}\right),
\end{gathered}
$$

where $\omega_{\sigma}$ is spinor satisfying $(\vec{n} \cdot \vec{\sigma}) \omega_{\sigma}=\sigma \cdot \omega_{\sigma}$ and $\sigma$ takes values \pm 1 .

The $H$ and $h$ parameters are then, for scalar case:

$$
H^{\mu j}=h^{\mu j}=1,
$$

for spin 1:

$$
\begin{aligned}
& \left\{\begin{array}{rl}
H_{\vec{k}, 0}^{\mu j} & =\frac{\epsilon_{\mu}(k) \epsilon_{j}(k)-k^{2}}{m_{\mu} m_{j}} \\
h_{\vec{k}, 0}^{\mu j} & =\frac{\epsilon_{\mu}(k) \epsilon_{j}(k)+k^{2}}{m_{\mu} m_{j}}
\end{array}, \sigma=0\right. \\
& H_{\vec{k}, \pm}^{\mu j}=h_{\vec{k}, \pm}^{\mu j}=1, \sigma= \pm 1 \text {; }
\end{aligned}
$$

and for spin 1/2:

$$
\begin{gathered}
H_{\vec{k}, \sigma}^{\mu j}=\sqrt{\left(\epsilon_{\mu}(k)+m_{\mu}\right)\left(\epsilon_{j}(k)+m_{j}\right)}+\sqrt{\left(\epsilon_{\mu}(k)-m_{\mu}\right)\left(\epsilon_{j}(k)-m_{j}\right)}, \\
h_{\vec{k}, \sigma}^{\mu j}=\sigma\left(\sqrt{\left(\epsilon_{\mu}(k)-m_{\mu}\right)\left(\epsilon_{j}(k)+m_{j}\right)}-\sqrt{\left(\epsilon_{\mu}(k)+m_{\mu}\right)\left(\epsilon_{j}(k)-m_{j}\right)}\right) .
\end{gathered}
$$

\section{Appendix C. Applications of General Quantum Field Theory of Flavor Mixing}

Appendix C.1. Vector Meson Mixing (S=1) for Two Flavors

We now consider the unitary mixing of two fields with spin 1 (vector mesons). $U(2)$ parametrization consists of four parameters: three phases that can be absorbed in the phase redefinition of fields and one essential real angle that is left, so that

$$
U=\left(\begin{array}{cc}
\cos (\theta) & \sin (\theta) \\
-\sin (\theta) & \cos (\theta)
\end{array}\right)
$$

Using Appendix B, we define $\gamma_{\mu i}^{ \pm}=\frac{1}{2}\left(\sqrt{\frac{\epsilon_{\mu}}{\epsilon_{i}}} \pm \sqrt{\frac{\epsilon_{i}}{\epsilon_{\mu}}}\right)$ for $\sigma= \pm 1$ and

$$
\begin{aligned}
& \gamma_{\mu i}^{+}=\frac{1}{2} \frac{\epsilon_{\mu} \epsilon_{i}-k^{2}}{m_{\mu} m_{i}}\left(\sqrt{\frac{\epsilon_{\mu}}{\epsilon_{i}}}+\sqrt{\frac{\epsilon_{i}}{\epsilon_{\mu}}}\right), \\
& \gamma_{\mu i}^{-}=\frac{1}{2} \frac{k^{2}+\epsilon_{\mu} \epsilon_{i}}{m_{\mu} m_{i}}\left(\sqrt{\frac{\epsilon_{\mu}}{\epsilon_{i}}}-\sqrt{\frac{\epsilon_{i}}{\epsilon_{\mu}}}\right)
\end{aligned}
$$


for $\sigma=0$. For the free-field mass parameters $m_{\mu}, \gamma_{12}^{+}=\gamma_{21}^{+}=\gamma_{+}, \gamma_{12}^{-}=-\gamma_{21}^{-}=\gamma_{-}$.

The ladder mixing matrices $\alpha$ and $\beta$ are given by

$$
\begin{aligned}
& \alpha=\left(\begin{array}{cc}
\cos (\theta) & \gamma+\sin (\theta) \\
-\gamma+\sin (\theta) & \cos (\theta) \\
0 & \gamma_{-} \sin (\theta) \\
-\gamma_{-} \sin (\theta) & 0
\end{array}\right),
\end{aligned}
$$

For the flavor charge oscillations we then obtain the result that is independent from the mass parametrization;

$$
\begin{gathered}
Q_{111}=1+\sin ^{2}(2 \theta)\left(\gamma_{-}^{2} \sin ^{2}\left(\frac{\Omega_{12} t}{2}\right)-\gamma_{+}^{2} \sin ^{2}\left(\frac{\omega_{12} t}{2}\right)\right), \\
Q_{121}=\sin ^{2}(2 \theta)\left(\gamma_{+}^{2} \sin ^{2}\left(\frac{\omega_{12} t}{2}\right)-\gamma_{-}^{2} \sin ^{2}\left(\frac{\Omega_{12} t}{2}\right)\right) .
\end{gathered}
$$

We see that this result, with an exception of greater complexity of $\gamma_{ \pm}$, is identical to the case of spin $0[35,36]$. According to the above theory, in fact, this should be expected for the two-flavor mixing with any integer spin. For $S=1$ we see that an essential difference from the scalar/pseudoscalar meson mixing, such as the complication of momentum dependence of $\gamma_{ \pm}$, occurs only for the mixing of longitudinally polarized particles. The mixing of transverse components is essentially the same as in the case of spin-zero particles.

The details of non-equal time commutators are given by

$$
\begin{aligned}
& F=\left\{\begin{array}{l}
e^{-i \epsilon_{1} t} \cos ^{2}(\theta)+e^{-i \epsilon_{2} t} \gamma_{+}^{2} \sin ^{2}(\theta)-e^{i \epsilon_{2} t} \gamma_{-}^{2} \sin ^{2}(\theta) ; \quad \gamma_{+} \sin (\theta) \cos (\theta)\left(e^{-i \epsilon_{2} t}-e^{-i \epsilon_{1} t}\right) \\
\gamma+\sin (\theta) \cos (\theta)\left(e^{-i \epsilon_{2} t}-e^{-i \epsilon_{1} t}\right) ; \quad e^{-i \epsilon_{2} t} \cos ^{2}(\theta)+e^{-i \epsilon_{1} t} \gamma_{+}^{2} \sin ^{2}(\theta)-e^{i \epsilon_{1} t} \gamma_{-}^{2} \sin ^{2}(\theta)
\end{array}\right\}, \\
& G=\left(\begin{array}{cc}
\gamma_{+} \gamma_{-} \sin ^{2}(\theta)\left(e^{-i \epsilon_{2} t}-e^{i \epsilon_{2} t}\right) & \gamma_{-} \sin (\theta) \cos (\theta)\left(e^{-i \epsilon_{1} t}-e^{i \epsilon_{2} t}\right) \\
\gamma_{-} \sin (\theta) \cos (\theta)\left(e^{-i \epsilon_{2} t}-e^{i \epsilon_{1} t}\right) & \gamma_{+} \gamma_{-} \sin ^{2}(\theta)\left(e^{i \epsilon_{1} t}-e^{-i \epsilon_{1} t}\right)
\end{array}\right) .
\end{aligned}
$$

The condensates of free-field particles are

$$
Z_{1}^{\prime}=Z_{2}^{\prime}=\gamma_{-}^{2} \sin ^{2}(\theta)
$$

and the condensates of the flavor particles in the vacuum are

$$
\begin{aligned}
& Z_{1}=4 \gamma_{-}^{2} \sin ^{2}(\theta)\left(\cos ^{2}(\theta) \sin ^{2}\left(\frac{\Omega_{12} t}{2}\right)+\gamma_{+}^{2} \sin ^{2}(\theta) \sin ^{2}\left(\frac{\Omega_{22} t}{2}\right)\right), \\
& Z_{2}=4 \gamma_{-}^{2} \sin ^{2}(\theta)\left(\cos ^{2}(\theta) \sin ^{2}\left(\frac{\Omega_{12} t}{2}\right)+\gamma_{+}^{2} \sin ^{2}(\theta) \sin ^{2}\left(\frac{\Omega_{11} t}{2}\right)\right) .
\end{aligned}
$$

The flavor vacuum structure is defined by the matrix $\hat{Z}$ :

$$
\hat{Z}=\frac{-1}{\left(\cos ^{2}(\theta)+\gamma_{+}^{2} \sin ^{2}(\theta)\right)}\left(\begin{array}{cc}
-\gamma_{+} \gamma_{-} \sin ^{2}(\theta) & \gamma_{-} \cos (\theta) \sin (\theta) \\
\gamma_{-} \cos (\theta) \sin (\theta) & \gamma_{+} \gamma_{-} \sin ^{2}(\theta)
\end{array}\right)
$$

with the normalization constant being $\mathcal{Z}=\left(1-\frac{\gamma_{-}^{2} \sin ^{2}(\theta)}{\cos ^{2}(\theta)+\gamma_{+}^{2} \sin ^{2}(\theta)}\right)^{-1}=1+\gamma_{-}^{2} \sin ^{2}(\theta)$. One can see that $\frac{1}{\mathcal{Z}}$ as function of $\gamma_{-}^{2} \sin ^{2}(\theta)$ has a pole at imaginary $\gamma_{-} \sin (\theta)=i$, so that the perturbative expansion in $\gamma_{-}^{2} \sin ^{2}(\theta)$ would have a finite convergence radius.

The time evolution of the flavor particle number (if \#1 was emitted) is given by:

$$
\begin{gathered}
N_{111}=1+\sin ^{2}(\theta)\left\{8 \gamma_{-}^{2} \cos ^{2}(\theta) \sin ^{2}\left(\frac{\Omega_{12} t}{2}\right)-4 \gamma_{+}^{2} \cos ^{2}(\theta) \sin ^{2}\left(\frac{\omega_{12} t}{2}\right)\right. \\
\left.+8 \gamma_{+}^{2} \gamma_{-}^{2} \sin ^{2}(\theta) \sin ^{2}\left(\frac{\Omega_{22} t}{2}\right)\right\}, \\
\bar{N}_{111}=4 \gamma_{-}^{2} \sin ^{2}(\theta)\left(2 \gamma_{+}^{2} \sin ^{2}(\theta) \sin ^{2}\left(\frac{\Omega_{22} t}{2}\right)+\cos ^{2}(\theta) \sin ^{2}\left(\frac{\Omega_{12} t}{2}\right)\right),
\end{gathered}
$$




$$
\begin{gathered}
N_{121}=\sin ^{2}(\theta)\left\{4 \gamma_{+}^{2} \cos ^{2}(\theta) \sin ^{2}\left(\frac{\omega_{12} t}{2}\right)+4 \gamma_{-}^{2} \cos ^{2}(\theta) \sin ^{2}\left(\frac{\Omega_{12} t}{2}\right)\right. \\
+4 \gamma_{+}^{2} \gamma_{-}^{2} \sin ^{2}(\theta) \sin ^{2}\left(\frac{\Omega_{11} t}{2}\right) \\
\bar{N}_{121}=4 \gamma_{-}^{2} \sin ^{2}(\theta)\left(2 \cos ^{2}(\theta) \cos ^{2}\left(\frac{\Omega_{12} t}{2}\right)+\gamma_{+}^{2} \sin ^{2}(\theta) \sin ^{2}\left(\frac{\Omega_{11} t}{2}\right)\right) .
\end{gathered}
$$

Also we note that the scalar and pseudoscalar case follows immediately from the above presentation when $\gamma_{\mu i}^{ \pm}=\frac{1}{2}\left(\sqrt{\frac{\epsilon_{\mu}}{\epsilon_{i}}} \pm \sqrt{\frac{\epsilon_{i}}{\epsilon_{\mu}}}\right)$. In this respect, the spin-zero mixing is equivalent to the mixing of transverse components of vector fields, described by Equations (A28), (A29), (A33) and (A34). These results are in accord with [35,36].

\section{Appendix C.2. Fermion Mixing $(S=1 / 2)$ for Two Flavors}

We also present here the calculations for the $S=1 / 2$ case. For the consistent notation with the previous works $[30,82]^{2}$, we define

$$
\begin{aligned}
U & =\frac{\sqrt{\left(\epsilon_{1}+m_{1}\right)\left(\epsilon_{2}+m_{2}\right)}+\sqrt{\left(\epsilon_{1}-m_{1}\right)\left(\epsilon_{2}-m_{2}\right)}}{2 \sqrt{\epsilon_{1} \epsilon_{2}}}, \\
V & =\sigma \frac{\sqrt{\left(\epsilon_{1}-m_{1}\right)\left(\epsilon_{2}+m_{2}\right)}-\sqrt{\left(\epsilon_{1}+m_{1}\right)\left(\epsilon_{2}-m_{2}\right)}}{2 \sqrt{\epsilon_{1} \epsilon_{2}}} .
\end{aligned}
$$

The charge fluctuations are then given by

$$
\begin{gathered}
Q_{111}=1-\sin ^{2}(2 \theta)\left(U^{2} \sin ^{2}\left(\frac{\omega_{12} t}{2}\right)+V^{2} \sin ^{2}\left(\frac{\Omega_{12} t}{2}\right)\right), \\
Q_{121}=\sin ^{2}(2 \theta)\left(U^{2} \sin ^{2}\left(\frac{\omega_{12} t}{2}\right)+V^{2} \sin ^{2}\left(\frac{\Omega_{12} t}{2}\right)\right)
\end{gathered}
$$

and the ladder mixing matrices are

$$
\begin{gathered}
\alpha=\left(\begin{array}{cc}
\cos (\theta) & U \sin (\theta) \\
-U \sin (\theta) & \cos (\theta)
\end{array}\right), \\
\beta=\left(\begin{array}{cc}
0 & V \sin (\theta) \\
V \sin (\theta) & 0
\end{array}\right),
\end{gathered}
$$

which are the same with [30,82].

We can give more details on the fermion mixing dynamics. The non-equal time anticommutators are given by

$$
\begin{array}{r}
F=\left\{\begin{array}{r}
e^{-i \epsilon_{1} t} \cos ^{2}(\theta)+e^{-i \epsilon_{2} t} U^{2} \sin ^{2}(\theta)+e^{i \epsilon_{2} t} V^{2} \sin ^{2}(\theta) ; \\
U \sin (\theta) \cos (\theta)\left(e^{-i \epsilon_{2} t}-e^{-i \epsilon_{1} t}\right) ; \quad e^{-i \epsilon_{2} t} \cos ^{2}(\theta)+e^{-i \epsilon_{1} t} U^{2} \sin ^{2}(\theta)+e^{i \epsilon_{1} t} V^{2} \sin ^{2}(\theta)
\end{array}\right\}, \\
G=\left(\begin{array}{cc}
U V \sin ^{2}(\theta)\left(e^{-i \epsilon_{2} t}-e^{i \epsilon_{2} t}\right) & V \sin (\theta) \cos (\theta)\left(e^{-i \epsilon_{1} t}-e^{i \epsilon_{2} t}\right) \\
V \sin (\theta) \cos (\theta)\left(e^{-i \epsilon_{2} t}-e^{i \epsilon_{1} t}\right) & U V \sin ^{2}(\theta)\left(e^{i \epsilon_{1} t}-e^{-i \epsilon_{1} t}\right)
\end{array}\right) .
\end{array}
$$

The condensates of the free-field particles are

$$
Z_{1}^{\prime}=Z_{2}^{\prime}=V^{2} \sin ^{2}(\theta)
$$

and the condensates of the flavor particles are

$$
\begin{aligned}
& Z_{1}=4 V^{2} \sin ^{2}(\theta)\left(\cos ^{2}(\theta) \sin ^{2}\left(\frac{\Omega_{12} t}{2}\right)+U^{2} \sin ^{2}(\theta) \sin ^{2}\left(\frac{\Omega_{22} t}{2}\right)\right), \\
& Z_{2}=4 V^{2} \sin ^{2}(\theta)\left(\cos ^{2}(\theta) \sin ^{2}\left(\frac{\Omega_{12} t}{2}\right)+U^{2} \sin ^{2}(\theta) \sin ^{2}\left(\frac{\Omega_{11} t}{2}\right)\right) .
\end{aligned}
$$

2 In our notation $U=\gamma_{+}, V=\gamma_{-}$. 
The vacuum structure is defined by the matrix $\hat{Z}$ :

$$
\hat{Z}=\frac{-1}{\cos ^{2}(\theta)+U^{2} \sin ^{2}(\theta)}\left(\begin{array}{cc}
-U V \sin ^{2}(\theta) & V \cos (\theta) \sin (\theta) \\
V \cos (\theta) \sin (\theta) & U V \sin ^{2}(\theta)
\end{array}\right)
$$

with the normalization constant being $\mathcal{Z}=\frac{1}{\cos ^{2}(\theta)+U^{2} \sin ^{2}(\theta)}=\frac{1}{1-V^{2} \sin ^{2}(\theta)}$.

The time evolution of the flavor particle number (if \#1 was emitted) is then given by:

$$
\begin{gathered}
N_{111}=1-4 U^{2} \sin ^{2}(\theta) \cos ^{2}(\theta) \sin ^{2}\left(\frac{\omega_{12} t}{2}\right), \\
\bar{N}_{111}=4 V^{2} \sin ^{2}(\theta) \cos ^{2}(\theta) \sin ^{2}\left(\frac{\Omega_{12} t}{2}\right), \\
N_{121}=4 \sin ^{2}(\theta)\left\{U^{2} \cos ^{2}(\theta) \sin ^{2}\left(\frac{\omega_{12} t}{2}\right)+V^{2} \cos ^{2}(\theta) \sin ^{2}\left(\frac{\Omega_{12} t}{2}\right)\right. \\
\left.+U^{2} V^{2} \sin ^{2}(\theta) \sin ^{2}\left(\frac{\Omega_{11} t}{2}\right)\right\}, \\
\bar{N}_{121}=4 U^{2} V^{2} \sin ^{4}(\theta) \sin ^{2}\left(\frac{\Omega_{11} t}{2}\right) .
\end{gathered}
$$

\section{Appendix C.3. Boson and Fermion Mixing for Three Flavors}

Mixing of three and more flavors can be considered in full analogy to the calculations presented above using our unified formulation. Due to growing complexity of $S U(N)$ general parametrization for larger $N$, it makes better sense to consider such cases in numerical form, applying our formalism to an explicitly given mixing matrix. Nonetheless, some results for mixing in $S U(3)$ are known in the literature in Standard and Wolfenstein parametrization [84]. Due to the size of the relevant formulas, we do not find it possible to explicitly list these results here. The interested reader should look at the relevant publications $[37,38]$. When treating such problem in general, it may be useful to keep in mind a few comments immediately following from the general formalism. In particular, mixing of $\mathrm{N}$-flavors have similar structure for any spin with spin-information being encoded implicitly in $\alpha_{\mu i}, \beta_{\mu i}$ mixing parameters. Once such result is written in terms of $\alpha$ and $\beta$, immediate generalization to other spins can be obtained. Furthermore, there exist close parallels between results for bosons and fermions. If one goes from the fermion to boson case, the sign of $\beta^{2}$ terms shall be changed and $\alpha, \beta$ parameters should be reinterpreted according to the spin change. For these reasons, in fact, it is sufficient to know only one case of mixing, say fermions, in given $S U(N)$ parametrization to extract the general result. In particular, Reference [38] presents $S U(3)$ calculations in standard parametrization, which can be appropriately generalized to describe a boson case. Reference [37] considers both the boson and fermion case for Wolfenstein parametrization of $S U(3)$, although one shall keep in mind that this parametrization is only an approximate description of a $S U(3)$ matrix.

\section{References}

1. Cheng, T.; Li, L. Gauge Theory of Elementary Particle Physics; Oxford University Press: Oxford, UK, 1984.

2. Marshak, R.E. Conceptual Foundations of Modern Particle Physics; World Scientific: Singapore, 1993.

3. Bilenky, S.M.; Pontecorvo, B. Lepton mixing and neutrino oscillations. Phys. Rep. 1978, 41, 225. [CrossRef]

4. Bilenky, S.M.; Petcov, S.T. Massive neutrinos and neutrino oscillations. Rev. Mod. Phys. 1987, 59, 671. [CrossRef]

5. Fukuda, Y.; Hayakawa, T.; Ichihara, E.; Inoue, K.; Ishihara, K.; Ishino, H.; Itow, Y.; Kajita, T.; Kameda, J.; Kasuga, S.; et al. (Super-Kamiokande Collaboration) Evidence for Oscillation of Atmospheric Neutrinos. Phys. Rev. Lett. 1998, 81, 1562. [CrossRef]

6. Hatakeyama, S.; Hara, T.; Fukuda, Y.; Hayakawa, T.; Inoue, K.; Ishihara, K.; Ishino, H.; Joukou, S.; Kajita, T.; Kasuga, S.; et al (Kamiokande Collaboration) Measurement of the Flux and Zenith-Angle Distribution of Upward Through-Going Muons in Kamiokande II + III. Phys. Rev. Lett. 1998, 81, 2016. [CrossRef]

7. Kobayashi, M.; Maskawa, T. CP-Violation in the Renormalizable Theory of Weak Interaction. Prog. Theor. Phys. 1973, $49,652$. [CrossRef]

8. Cabibbo, N. Unitary Symmetry and Leptonic Decays. Phys. Rev. Lett. 1963, 10, 531. [CrossRef] 
9. Koshiba, M. The neutrino oscillations as observed by Kamiokandes and by Super-Kamiokande. In From the Planck Length to the Hubble Radius, Proceedings of the International School of Subnuclear Physics, Erice, Italy, 29 August-7 September, 1998; World Scientific: Singapore, 2000; pp. 170-198.

10. Fukuda, S.; Fukuda, Y.; Ishitsuka, M.; Itow, Y.; Kajita, T.; Kameda, J.; Kaneyuki, K.; Kobayashi, K.; Koshio, Y.; Miura, M.; et al. (Super-Kamiokande collaboration) Constraints on Neutrino Oscillations using 1258 days of Super-Kamiokande solar neutrino data. Phys. Rev. Lett. 2001, 86, 5656. [CrossRef]

11. Ahmad, Q.R.; Allen, R.C.; Andersen, T.C.; Anglin, J.D.; Bühler, G.; Barton, J.C.; Beier, E.W.; Bercovitch, M.; Bigu, J.; Biller, S.; et al. (SNO collaboration) Measurement of the Rate of $v_{e}+d \rightarrow p+p+e^{-}$Interactions Produced by ${ }^{8} B$ Solar Neutrinos at the Sudbury Neutrino Observatory. Phys. Rev. Lett. 2001, 87, 071301. [CrossRef]

12. Ahmad, Q.R.; Allen, R.C.; Andersen, T.C.; Anglin, J.D.; Barton, J.C.; Beier, E.W.; Bercovitch, M.; Bigu, J.; Biller, S. D.; Black, R.A.; et al. (SNO collaboration) Direct Evidence for Neutrino Flavor Transformation from Neutral-Current Interactions in the Sudbury Neutrino Observatory. Phys. Rev. Lett. 2002, 89, 011301. [CrossRef]

13. Eguchi, K.; Enomoto, S.; Furuno, K.; Goldman, J.; Hanada, H.; Ikeda, H.; Ikeda, K.; Inoue, K.; Ishihara, K.; Itoh, W.; et al. (KamLAND Collaboration) First Results from KamLAND: Evidence for Reactor Antineutrino Disappearance. Phys. Rev. Lett. 2003, 90, 021802. [CrossRef]

14. Ahn, M.H.; Aoki, S.; Bhang, H.; Boyd, S.; Casper, D.; Choi, J.H.; Fukuda, S.; Fukuda, Y.; Gajewski, W.; Hara, T.; et al. (K2K Collaboration) Indications of neutrino oscillation in a $250 \mathrm{~km}$ long-baseline experiment. Phys. Rev. Lett. 2003, $90,041801$. [CrossRef] [PubMed]

15. Mohapatra, R.; Pal, P. Massive Neutrinos in Physics and Astrophysics; World Scientific: Singapore, 1991.

16. Bahcall, J.N. Neutrino Astrophysics; Cambridge University Press: Cambridge, UK, 1989.

17. Oberauer, L.; von Feilitzsch, F. Neutrino oscillations. Rep. Prog. Phys. 1992, 55, 1093. [CrossRef]

18. Kim, C.W.; Pevsner, A. Neutrinos in Physics and Astrophysics. Contemporary Concepts in Physics; Harwood Academic: Chur, Switzerland, 1993; Volume 8.

19. Pascoli, S.; Petcov, S. The SNO solar neutrino data, neutrinoless double beta-decay and neutrino mass spectrum. Phys. Lett. 2002, B544, 239. [CrossRef]

20. Nikolić, H. Generalizations of normal ordering and applications to quantization in classical backgrounds. Gen. Relativ. Grav. 2005. 37, 297311. [CrossRef]

21. McGregor, G.A. First Results from the Sudbury Neutrino Observatory. arXiv 2002, arXiv:nucl-ex/0205006. Available online:https: / / arxiv.org/abs/nucl-ex/0205006 (accessed on 25 February 2021).

22. Dazeley, S. An Update on Progress at KamLAND. arXiv 2002, arXiv:hep-ex/0205041. Available online: https: / / arxiv.org/abs/hep-ex/0205041 (accessed on 25 February 2021).

23. Christenson, J.H.; Cronin, J.W.; Fitch, V.L.; Turlay, R. Evidence for the $2 \pi$ Decay of the $K_{2}^{0}$ Meson. Phys. Rev. Lett. 1964, $13,138$. [CrossRef]

24. Ji, C.-R.; Choi, H.M. For a recent theoretical overview. Nucl. Phys. 2000, B90, 93. [CrossRef]

25. Blasone, M.; Henning, P.A.; Vitiello, G. The exact formula for neutrino oscillations. Phys. Lett. 1999, B451, 140. [CrossRef]

26. Blasone, M. New Results in the Physics of Neutrino Oscillations. In From the Planck Length to the Hubble Radius, Proceedings of the International School of Subnuclear Physics, Erice, Italy, 29 August-7 September, 1998; World Scientific: Singapore, 2000 ; pp. 584-593.

27. Binger, M.; Ji, C.-R. Quantum field theory of meson mixing. Phys. Rev. D 1999, 60, 056005. [CrossRef]

28. Fujii, K.; Habe, C.; Yabuki, T. Note on the field theory of neutrino mixing. Phys. Rev. 1999, D59, 113003. [CrossRef]

29. Fujii, K.; Habe, C.; Yabuki, T. Remarks on flavor-neutrino propagators and oscillation formulas. Phys. Rev. D 2001, 64, 013011. [CrossRef]

30. Blasone, M.; Vitiello, G. Quantum Field Theory of Fermion Mixing. Ann. Phys. 1995, 244, 283-311. [CrossRef]

31. Bogoliubov, N.; Shirkov, D. Introduction to the Theory of Quantized Fields; John Wiley: New York, NY, USA, 1980.

32. Itzykson, C.; Zuber, J. Quantum Field Theory; McGraw-Hill Co.: New York, NY, USA, 1980.

33. Blasone, M.; Capolupo, A.; Vitiello, G. Understanding Flavor Mixing in Quantum Field Theory. In Flavor Physics, Proceedings of the ICFP2001, Zhang-Jia-Jie, China, 31 May-6 June 2001; World Scientific: Singapore, 2002; pp. 425-433.

34. Blasone, M.; Capolupo, A.; Vitiello, G. Comment on "Remarks on flavor-neutrino propagators and oscillation formulae". arXiv 2001, arXiv:hep-ph/0107183. Available online: https://arxiv.org/abs/hep-ph/0107183 (accessed on 25 February 2021).

35. Blasone, M.; Capolupo, A.; Romei, O.; Vitiello, G. Quantum field theory of boson mixing. Phys. Rev. D 2001, 63, 125015. [CrossRef]

36. Ji, C.-R.; Mishchenko, Y. Nonperturbative vacuum effect in the quantum field theory of meson mixing. Phys. Rev. D 2001, 64, 076004. [CrossRef]

37. Ji, C.-R.; Mishchenko, Y. General theory of quantum field mixing. Phys. Rev. 2002, D65, 096015. [CrossRef]

38. Blasone, M.; Capolupo, A.; Vitiello, G. Quantum field theory of three flavor neutrino mixing and oscillations with CP violation. Phys. Rev. D 2002, 66, 025033. [CrossRef]

39. Hannabuss, K.C.; Latimer, D.C. Fermion mixing in quasifree states. J. Phys. A 2003, 36, L69. [CrossRef]

40. Hannabuss, K.C.; Latimer, D.C. The quantum field theory of fermion mixing. J. Phys. A. 2000, 33, 1369. [CrossRef]

41. Itzykson, C. Remarks on boson commutation rules. Comm. Math. Phys. 1967, 4, 92. [CrossRef]

42. Bargmann, V. Analytic Methods in Mathematical Physics; Gilbert, E.P., Newton, R.G.; Gordon and Breach: New York, NY, USA, 1970; p. 271. 
43. Deenen, J.; Moshinsky, M.; Seligman, T.H. Canonical transformations to action and angle variables and their representations in quantum mechanics: III. The general problem. Ann. Phys. 1980, 127, 458. [CrossRef]

44. Garrod, C. Hamiltonian Path-Integral Methods. Rev. Mod. Phys. 1966, 38, 483. [CrossRef]

45. Goldstein, H. Classical Mechanics; Addison-Wesley Press: Cambridge, UK, 1950; p. 399

46. Anderson, A. Canonical Transformations in Quantum Mechanics. Ann. Phys. 1994, 232, 292. [CrossRef]

47. Mello, P.A.; Moshinsky, M.J. Nonlinear canonical transformations and their representations in quantum mechanics. Math. Phys. 1975, 16, 2017. [CrossRef]

48. Moshinsky, M. Groups in Physics; Les Presses de K'Universite de Montreal: Montreal, QC, Canada, $1979 ;$ p. 48.

49. Anderson, A. Quantum canonical transformations. Physical equivalence of quantum theories. Phys. Lett. B, 1993, $305,67$. [CrossRef]

50. Anderson, A. Quantum canonical transformations and integrability. Beyond unitary transformations. Phys. Lett. B, 1993, 319, 157. [CrossRef]

51. Dirac, P.A.M. The Principles of Quantum Mechanics, 4th ed.; Oxford Univ. Press: Oxford, UK, 1958.

52. Weyl, H. The Theory of Groups and Quantum Mechanics, 2nd ed.; Dover: New York, NY, USA, 1950; p. 1931.

53. Kim, J.-H.; Lee, H.-W. Canonical transformations and the Hamilton-Jacobi theory in quantum mechanics. Can. J. Phys. 1999, 77, 411. [CrossRef]

54. Hietarinta, J. Quantum canonical transformations as integral transformations. Phys. Rev. D 1982, 25, 210. [CrossRef]

55. Ghandour, G.I. Effective generating functions for quantum canonical transformations. Phys. Rev. D 1987, 35, 1289. [CrossRef] [PubMed]

56. Lee, H.; l'Yi, W.S. Non-Hermitian techniques of canonical transformations in quantum mechanics. Phys. Rev. A 1995, 51, 982. [CrossRef]

57. Kapoor, A.K. Quantization in nonlinear coordinates via Hamiltonian path integrals. Phys. Rev. D 1984, 29, 2339. [CrossRef]

58. Kapoor, A.K. Quantization in nonlinear coordinates via Hamiltonian path integrals. II. Phys. Rev. D 1984, 30, 1750. [CrossRef]

59. Blau, M.; Keski-Vakkuri, E.; Niemi, A. Path integrals and geometry of trajectories. Phys. Lett. B 1990, 246, 92. [CrossRef]

60. Bogoliubov, N.N. On the theory of superfluidity. J. Phys. (USSR) 1947, 11, 23.

61. Bogoliubov, N.N. On a new method in the theory of superconductivity. Nuovo Cim. 1958, 7, 794. [CrossRef]

62. Valatin, J.G. Comments on the theory of superconductivity. Nuovo Cim. 1958, 7, 843. [CrossRef]

63. Fetter, A. L.; Walecka, J. D. Quantum Theory of Many-particle Systems; McGraw-Hill: New York, NY, USA, 1980.

64. Bicudo, P.; Ribeiro, J.E. $\pi, K, \rho, \phi$ as $q \bar{q}$ bound states of the Salpeter equation in a ${ }^{3} P_{0}$ condensed vacuum. Phys. Rev. D 1990, 42 , 1625. [CrossRef] [PubMed]

65. Bicudo, P.; Ribeiro, J.; Rodrigues, J. $K-N$ S channel $I=1,0$ phase shifts as a direct measure of chiral condensation Phys. Rev. C 1995, 52, 2144.

66. Bicudo, P.J.; Ribeiro, J.E.; Nefediev, A. V. Vacuum replicas in QCD. Phys. Rev. D 2002, 65, 085026. [CrossRef]

67. Szczepaniak, A.P.; Swanson, E.S. Nonperturbative renormalization and the QCD vacuum. Phys. Rev. D 2000, 62, 094027. [CrossRef]

68. Kapitza, P. Viscosity of Liquid Helium below the $\lambda$-Point. Nature 1938, 141, 74. [CrossRef]

69. Landau, L.D. The theory of superfuidity of helium II. J. Phys. (USSR) 1941, 5, 71.

70. Lifshitz, E.M.; Pitaevskii, L.P. Statistical Physics; Pergamon: Oxford, UK, 1980.

71. Llanes-Estrada, F.J.; Cotanch, S.R. Relativistic many-body Hamiltonian approach to mesons. Nucl. Phys. A 2002, 697, 303; [CrossRef]

72. 't Hooft, G. A two-dimensional model for mesons. Nucl. Phys. 1974, B75, 461. [CrossRef]

73. Bars, I.; Green, M.B. Poincaré- and gauge-invariant two-dimensional quantum chromodynamics. Phys. Rev. D 1978, 17, 537. [CrossRef]

74. Li, M.; Wilets, L.; Birse, M.C. QCD 2 in the axial gauge. J. Phys. G Nucl. Phys. 1987, 13, 915. [CrossRef]

75. Ma, B.; Ji, C.-R. Interpolating $\mathrm{QCD}_{2}$ between the instant and front forms of relativistic dynamics. AIP Conf. Proc. 2020, 2249, 030009.

76. Kalashnikova, Y.S.; Nefed'ev, A.V. Two-dimensional QCD in the Coulomb gauge. Phys. Usp. 2002, 45, 347. [CrossRef]

77. Glozman, L.Y.; Sazonov, V.K.; Shifman, M.; Wagenbrunn, R.F. How chiral symmetry breaking affects the spectrum of the light-heavy mesons in the 't Hooft model. Phys. Rev. D 2012, 85, 094030. [CrossRef]

78. Jia, Y.; Liang, S.; Li, L.; Xiong, X. Solving the Bars-Green equation for moving mesons in two-dimensional QCD. JHEP 2017, 11, 151. [CrossRef]

79. Jia, Y.; Liang, S.; Xiong, X.; Yu, R. Partonic quasidistributions in two-dimensional QCD. Phys. Rev. D 2018, 98, 054011. [CrossRef]

80. Ring, P.; Schuck, P. The Nuclear Many Body Problem; Springer: New York, NY, USA, 1980.

81. Mattueck, R. A Guide to Feynman Diagrams in the Many Body Problem; Dover: New York, NY, USA, 1992.

82. Blasone, M.; Vitiello, G. Remarks on the neutrino oscillation formula. Phys. Rev. D 1999, 60, 111302. [CrossRef]

83. Ji, C.-R.; Mishchenko, Y. Time-to-space conversion in quantum field theory of flavor mixing. Ann. Phys. 2005, 315, 488. [CrossRef]

84. Wolfenstein, L. Parametrization of the Kobayashi-Maskawa Matrix. Phys. Rev. Lett. 1983, 51, 1945. [CrossRef] 